\title{
An ensemble of regulatory elements controls Runx3 spatiotemporal expression in subsets of dorsal root ganglia proprioceptive neurons
}

\author{
Elena Appel, ${ }^{1,7}$ Sarit Weissmann, ${ }^{1,5,7}$ Yehuda Salzberg, ${ }^{1,2,7}$ Kira Orlovsky, ${ }^{1}$ Varda Negreanu, ${ }^{1}$ \\ Michael Tsoory, ${ }^{3}$ Calanit Raanan, ${ }^{3}$ Ester Feldmesser, ${ }^{4}$ Yael Bernstein, ${ }^{1}$ Orit Wolstein, ${ }^{1,6}$ Ditsa Levanon, ${ }^{1}$ \\ and Yoram Groner ${ }^{1}$ \\ ${ }^{1}$ Department of Molecular Genetics, ${ }^{2}$ Department of Biomolecular Sciences, ${ }^{3}$ Department of Veterinary Resources, ${ }^{4}$ Life Science \\ Core Facilities, The Weizmann Institute of Science, Rehovot, 7610001, Israel
}

The Runx3 transcription factor is essential for development and diversification of the dorsal root ganglia (DRGs) TrkC sensory neurons. In Runx3-deficient mice, developing TrkC neurons fail to extend central and peripheral afferents, leading to cell death and disruption of the stretch reflex circuit, resulting in severe limb ataxia. Despite its central role, the mechanisms underlying the spatiotemporal expression specificities of Runx 3 in TrkC neurons were largely unknown. Here we first defined the genomic transcription unit encompassing regulatory elements (REs) that mediate the tissue-specific expression of Runx3. Using transgenic mice expressing BAC reporters spanning the Runx3 locus, we discovered three REs-dubbed R1, R2, and R3-that cross-talk with promoter-2 (P2) to drive TrkC neuron-specific Runx3 transcription. Deletion of single or multiple elements either in the BAC transgenics or by CRISPR/Cas9-mediated endogenous ablation established the REs' ability to promote and/or repress Runx3 expression in developing sensory neurons. Our analysis reveals that an intricate combinatorial interplay among the three REs governs Runx3 expression in distinct subtypes of TrkC neurons while concomitantly extinguishing its expression in non-TrkC neurons. These findings provide insights into the mechanism regulating cell type-specific expression and subtype diversification of TrkC neurons in developing DRGs.

[Keywords: Runx3 transcription factor; DRG TrkC proprioceptive neurons; long-range transcription regulatory elements; BAC transgenic mice; CRISPR/Cas9 enhancer mutants]

Supplemental material is available for this article.

Received October 1, 2016; revised version accepted November 16, 2016.

Runx3 is a member of the mammalian RUNX family of transcription factors (TFs), which are key gene expression regulators in several important developmental processes (Levanon and Groner 2004). During embryonic development, Runx3 expression is first detected at around embryonic day 11 (E11) in the dorsal root ganglia (DRGs) and at later stages in developing bones, whiskers, hair follicles, and hematopoietic cells (Levanon et al. 2001, 2011). Loss of Runx3 in these cell types impaired their function, leading to phenotypic defects (Inoue et al. 2002; Levanon et al. 2002, 2014; Yamashiro et al. 2002; Woolf et al. 2003; Bren-

Present addresses: ${ }^{5}$ Donald Danforth Plant Science Center, St. Louis, MO 63132, USA; ${ }^{6}$ Calimmune Pty. Ltd., Darlinghurst, New South Wales 2010, Australia.

${ }^{7}$ These authors contributed equally to this work.

Corresponding authors: yoram.groner@weizmann.ac.il, ditsa.levanon@ weizmann.ac.il

Article is online at http://www.genesdev.org/cgi/doi/10.1101/gad.291484. 116. Freely available online through the Genes \& Development Open Access option. ner et al. 2004; Fainaru et al. 2004; Raveh et al. 2005; Djuretic et al. 2007; Cruz-Guilloty et al. 2009; Naito and Taniuchi 2010; Dicken et al. 2013; Lotem et al. 2013; Bauer et al. 2015).

The DRGs include three main subclasses of sensory neurons distinguishable by their neurotrophin receptors: the nociceptive TrkA neurons, the mechanoceptive TrkB neurons, and the proprioceptive TrkC neurons. RUNX TFs play key roles in the post-mitotic diversification of these neurons into distinct sensory modalities (Lallemend and Ernfors 2012). Runx1 and Runx3 are differentially expressed in TrkA and TrkC neurons, respectively (Levanon et al. 2001, 2002; Inoue et al. 2002; Chen et al. 2006b; Kramer et al. 2006; Nakamura et al. 2008). Interestingly, Runx3, the phylogenetically most

(C) 2016 Appel et al. This article, published in Genes \& Development, is available under a Creative Commons License (Attribution-NonCommercial 4.0 International), as described at http://creativecommons.org/licenses/by-nc/4.0/. 
ancient mammalian RUNX (Bangsow et al. 2001; Levanon et al. 2003), regulates the neurogenesis of TrkC neurons (Inoue et al. 2002; Levanon et al. 2002; Chen et al. 2006a; Kramer et al. 2006) that are a major constituent of the simplest and most ancient neuronal circuit: the stretch reflex arc (Levanon et al. 2003; Sullivan et al. 2008). In the absence of Runx3, TrkC neurons are initially formed but fail to extend peripheral afferents and undergo apoptosis, leading to congenital ataxia (Levanon et al. 2002). The strict specificity to TrkC neurons implies that Runx3 expression is tightly regulated. However, little was known about the molecular mechanisms regulating the spatiotemporal expression of Runx3 in developing TrkC neurons. Here, we used reporter BAC transgenics and CRISPR/Cas9-mediated gene editing to demonstrate that TrkC neuron-specific Runx3 transcription is regulated by an intricate cross-talk between promoter-2 (P2) and three upstream regulatory elements (REs) that promote Runx3 expression in distinct TrkC neuron subtypes and extinguish it in non-TrkC neurons.

\section{Results}

A genomic region spanning $170 \mathrm{~kb}$ is required for authentic full-fledged Runx3 expression in developing mouse embryos

Runx3 belongs to a group of developmental TFs that are frequently regulated by promoter/enhancer cross-talk to establish their spatiotemporal expression specificity during embryogenesis (Buecker and Wysocka 2012; Cannavo et al. 2016). To define the entire transcriptional unit of Runx3, including the required REs, we collected six overlapping BACs that span $340 \mathrm{~kb}$ of the Runx3 locus and its $5^{\prime}$ and $3^{\prime}$ flanking regions (Fig. 1A; Supplemental Table S1). We then converted each BAC into a reporter construct by the in-frame insertion of $L a c Z$ or EGFP into Runx3 exon 3, which appears in all functional gene products (Fig. 1A; Bangsow et al. 2001). Using transient BAC transgenesis, we found that the overall expression pattern of the six BAC-LacZ reporter constructs faithfully recapitulated the previously well-documented pattern of Runx3 expression (Bangsow et al. 2001; Levanon et al. 2011). This analysis defined a genomic region of $\sim 170 \mathrm{~kb}$, contained in BAC-A and BAC-C, as required and sufficient for the specific spatiotemporal expression of Runx3 (Supplemental Fig. S1).

\section{$P 2$ is mandatory for Runx3 expression in DRG neurons}

Runx3 expression is mediated by two distinct promoters, designated P1 and P2 (Levanon et al. 2011). Analysis of Runx3 promoter-specific knock-in mice-i.e., P1 knockin $\left(\mathrm{P} 1^{\mathrm{AFP} /+}\right)$ and $\mathrm{P} 2$ knock-in $\left(\mathrm{P} 2^{\mathrm{GFP} /+}\right)$ (Supplemental Fig. S2)-revealed that, from E11 onward, the knock-in reporter gene and endogenous Runx3 were coexpressed in $\mathrm{P} 2{ }^{\mathrm{GFP} /+}$ but not in $\mathrm{P} 1^{\mathrm{AFP} /+}$ heterozygous mice (Fig. $1 \mathrm{~B}$, top panels). This observation demonstrates that Runx3 expression in TrkC neurons is mediated by $\mathrm{P} 2$. Accordingly, homozygous P2 ${ }^{\text {GFP/GFP }}$ mice developed severe limb atax- ia, whereas P1 ${ }^{\mathrm{AFP} / \mathrm{AFP}}$ mice did not. The ataxia in homozygous P2 $2^{\text {GFP/GFP }}$ mice was caused by the loss of Runx 3 in TrkC neurons as early as E11 (Fig. 1B, middle panels), recapitulating the Runx $3^{-/-}$phenotype (Levanon et al. 2002). In contrast, P1 ${ }^{\mathrm{AFP} / \mathrm{AFP}}$ mice phenotypically resembled wild-type mice and coexpressed endogenous Runx3 and TrkC at all embryonic stages (Fig. 1B, bottom panels). In Runx3-P2 ${ }^{\text {GFP/GFP }}$ mice lacking Runx3, TrkC expression was retained until E11.5 (Fig. 1B, middle panels), in agreement with a previous report that Runx3 is essential for maintenance of TrkC neurons but not for initial expression of TrkC (Kramer et al. 2006).

\section{Distantly located REs confer TrkC neuron-specific Runx3 expression}

Of the six BACs, only those that extend $5^{\prime}$ upstream of Runx3 (namely, BAC-E, BAC-C, and, to a lesser extent, BAC-A) conferred LacZ expression in E14.5 DRGs (Fig. 1A,C; Supplemental Table S1). In contrast, BAC-D, BAC-F, and BAC-B did not drive LacZ expression in DRGs, although they contained P2. These results show that P2 alone cannot drive Runx3 expression in DRG neurons and indicated that a genomic region upstream of BAC-B (boxed in Fig. 1A) contains DRG REs. Next, to identify conserved REs, we conducted a multiple species alignment using mVista (Frazer et al. 2004). The genomic sequences surrounding mouse Runx3 were compared with sequences from the orthologous regions in five vertebrates: humans, rats, chickens, frogs, and pufferfish (i.e., fugu) (Fig. 1A, bottom panel). Frogs and fish were included because Runx3 function in the sensory stretch reflex arc is phylogenetically ancient (Levanon et al. 2003; Sullivan et al. 2008), and therefore the regulatory sequences controlling its expression in TrkC neurons have likely been conserved over the course of evolution. The Vista analysis revealed 10 highly conserved noncoding elements (CNEs) within and surrounding Runx3 (Supplemental Table S2). Of these elements, four CNEs, marked $\mathrm{R} 1-\mathrm{R} 4$, were located in the presumable DRG regulatory region.

\section{Conserved elements R1, R2, and R3 are essential for Runx3 expression in TrkC neurons}

BAC-C-GFP that contains R1, R2, and R3 conferred reporter expression to most of the Runx3-positive cells along the DRGs and was therefore selected for further studies focusing on brachial DRGs (Fig. 1D, left). Deletion of these REs, yielding BAC-C-delR1,2,3-GFP mice (Supplemental Table S3), abolished reporter expression in developing TrkC neurons as early as E11.5 (Fig. 1D, right). This result indicates that these three REs are required for Runx3 expression in TrkC neurons. The essential role of these REs was further verified in mice carrying homozygous CRISPR/Cas9-mediated (CR) deletion $(\Delta)$ of the 25 -kb genomic region spanning R1-R3 (CR $\Delta \mathrm{R} 1,2,3 / \mathrm{CR} \Delta \mathrm{R} 1,2,3)$ (Supplemental Fig. S3; Supplemental Table S4), which developed severe ataxia, similar to Runx $3^{-/-}$mice (Levanon et al. 2002). In the compound heterozygous mice $\mathrm{CR} \Delta \mathrm{R} 1,2,3 / \mathrm{P} 2 \mathrm{GFP}$ 
Downloaded from genesdev.cshlp.org on April 26, 2023 - Published by Cold Spring Harbor Laboratory Press

Driving TrkC neuron-specific Runx3 expression

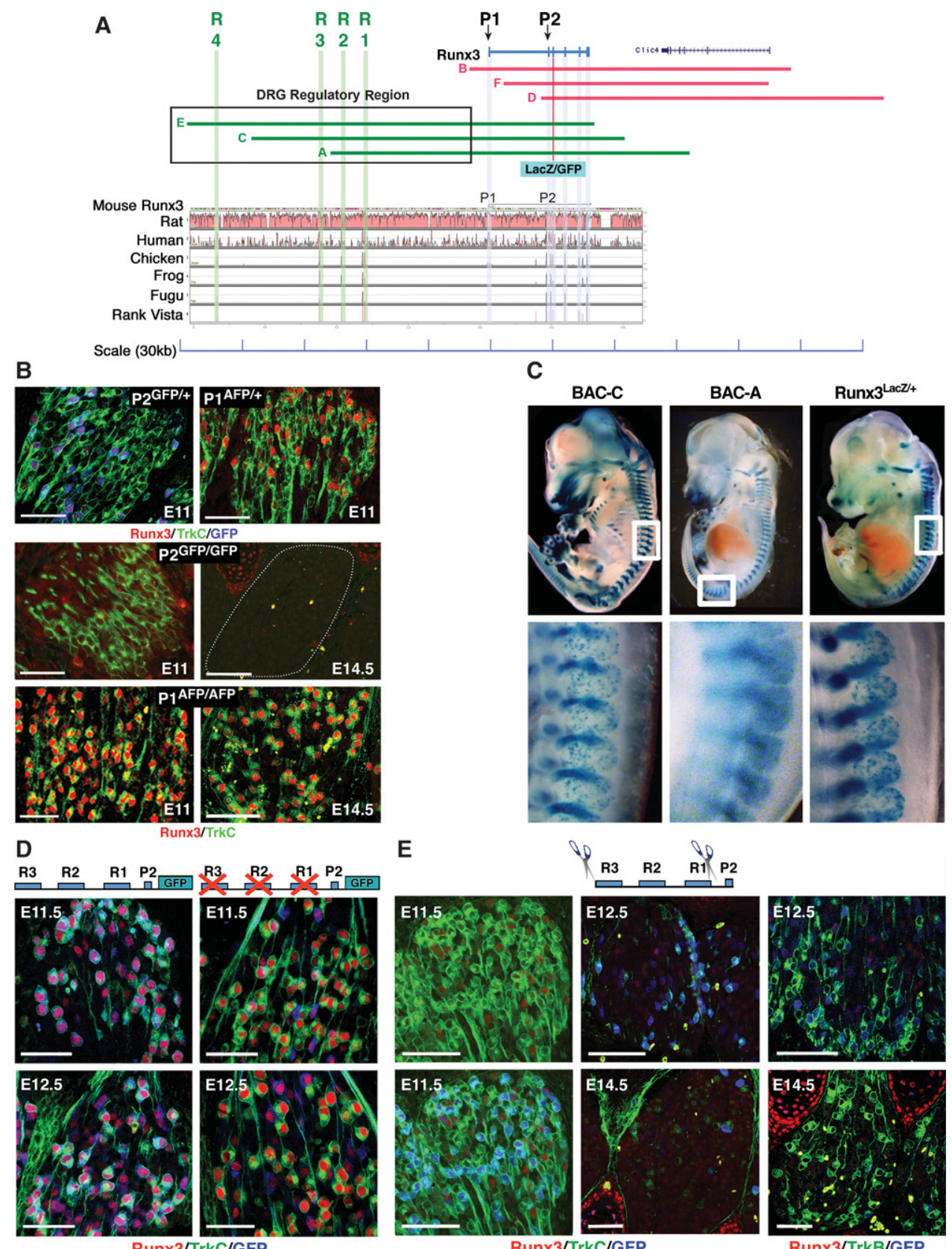

Figure 1. The Runx3 transcriptional unit: gene structure, REs, and DRG expression. (A, top panels) Schematic presentation of six BAC reporters marked as A, C, and E (green bars) and B, D, and F (red bars) (chromosome 4: 134,953,991-135,328,237; University of California at Santa Cruz [UCSC], mm10) spanning the Runx3 transcription unit. The blue box below the BACs represents the LacZ/GFP reporter inserted into the Runx3 coding region (see also Supplemental Table S1; Supplemental Fig. S1). (Bottom panel) Vista comparative analysis demonstrating the evolutionary conservation of the Runx3 transcriptional unit. The four REs—R1, R2, R3, and R4—are highlighted. (B) Runx3-P2 drives expression in developing DRG neurons. Immunofluorescence with anti-Runx3, anti-TrkC, and anti-GFP in E11 brachial DRGs of $\mathrm{P} 2{ }^{\mathrm{GFP} /+}$ (top left) and $\mathrm{P} 1^{\mathrm{AFP} /+}$ (top right). Expression of Runx3 and TrkC in P2 ${ }^{\mathrm{GFP} / \mathrm{GFP}}$ at E11 (middle left) and E14.5 (middle right). DRG is marked by a dashed line. (Middle right) Yellow dots represent blood cells. Runx3 expression in TrkC neurons of P1 ${ }^{\mathrm{AFP} / \mathrm{AFP}}$ at E11 (bottom left) and E14.5 (bottom right). (C) Expression of LacZ in BAC-C and BAC-A transgenics and Runx $3^{\text {LacZ/+ }}$ embryos at E14.5 (shown is the embryos' inner side). Boxed regions are enlarged in the bottom panels, demonstrating staining in DRGs. (D) BAC-C spans the three REs: R1, R2, and R3 (shown schematically above the top panels). GFP coexpression with TrkC and endogenous Runx3 in brachial DRG neurons at E11.5 (top left) and E12.5 (bottom left). Deletion of BAC-C REs (R1, R2, and R3) suspends GFP expression in TrkC/Runx3 neurons at E11.5 (top right) and E12.5 (bottom right). (E) CRISPR/Cas9-mediated deletion of the endogenous 25-kb region spanning R1, R2, and R3 (shown schematically above the panels). Expression of Runx3 and TrkC in $\triangle \mathrm{R} 1,2,3 / \mathrm{P} 2{ }^{\mathrm{GFP}}$ E1 1.5 embryos (top left) and same panel stained also with anti-GFP antibodies (bottom left). GFP derived from the P2-GFP allele serves as an internal control for TrkC neurons that lost Runx3. Expression of TrkC, Runx3, and GFP at E12.5 (top middle) and E14.5 (bottom middle). Expression of TrkB, Runx3, and GFP at E12.5 (top right) and E14.5 (bottom right). Note the expression of Runx3 at E14.5 in rib cartilage but not in DRGs. Bar, $50 \mu \mathrm{m}$. 
(Supplemental Fig. S3C), Runx3 expression in TrkC/GFP neurons was markedly lower at E11.5 (Fig. 1E, left). At E12.5, this Runx3 reduction was accompanied by a pronounced decrease in the number of TrkC neurons (Fig. 1E, top middle), with only a few TrkC/GFP neurons detected at E14.5 (Fig. 1E, bottom middle).

The loss of Runx 3 in TrkC neurons was associated with a gain in TrkB expression. Consequently, at E12.5, the number of TrkB-expressing neurons in CR $\Delta \mathrm{R} 1,2,3 / \mathrm{P} 2 \mathrm{GFP}$ DRGs increased and consisted of two populations (Fig. $1 \mathrm{E}$, top right). One population represented the conventional TrkB neurons, and the second, an unusual one, coexpressed TrkB and GFP. This latter population constituted TrkC neurons in which the loss of Runx3 led to TrkB expression. These observations correspond with previous findings that Runx3 represses $\operatorname{Trk} B$ and promotes Trk $C$ expression (Kramer et al. 2006; Inoue et al. 2007). The nearly complete loss of TrkC neurons at E14.5 in $\mathrm{CR} \Delta \mathrm{R} 1,2,3 / \mathrm{P} 2{ }^{\mathrm{GFP}}$ DRGs (Fig. 1E, bottom middle) was also evidenced by the marked reduction in neurons coexpressing TrkB and GFP, whereas the conventional TrkB neurons were maintained at a level comparable with that of wild-type DRGs (Fig. 1E, bottom right; data not shown). These results imply that TrkB could not functionally replace TrkC and thus did not rescue TrkC neurons from death due to loss of Runx3. The data also imply that the segregation between TrkB and TrkC neurons is not dependent on Runx3. Put together, the data demonstrate unequivocally that the three REs are essential for Runx3 expression, commencing from the earliest stages of TrkC neurogenesis.

\section{Cooperation between $R 1, R 2$, and $R 3$ promotes Runx3 expression at E11.5}

Next, we assessed the contribution of each of the three REs to TrkC neuron development through simultaneous analysis of BAC transgenic and compound CRISPR/ $\mathrm{P} 2{ }^{\mathrm{GFP}}$ mouse lines bearing single or double RE deletions (Supplemental Tables S3, S4). At E11.5, each single RE conferred Runx3 expression to most TrkC neurons (Fig. 2A-C). The intensity of Runx3 expression endowed by each single RE was significantly higher than in the absence of all three REs but lower compared with control (Fig. 2D). Similar results were obtained by analyzing lines of transgenic mice bearing RE-deleted BACs (Fig. 2E).

Interestingly, the loss of just $\mathrm{R} 1(\Delta \mathrm{R} 1)$ markedly reduced Runx3 expression level in TrkC neurons as compared with control, whereas deletion of $\mathrm{R} 3(\Delta \mathrm{R} 3)$ had no effect (Fig. 2B,D). These results indicate that, while at E11.5, the three REs are active and act cooperatively, R1 predominates. Taken together, these analyses establish that the onset of Runx3 expression in TrkC neurons at E11 is mediated by a synergism between the three REs.

\section{Developmental switch in RE usage at E12.5}

The unique contribution of each RE to Runx3 expression in TrkC neurons became evident at E12.5. Among single $\mathrm{RE}$ deletions, removal of $\mathrm{R} 1\left(\mathrm{CR} \Delta \mathrm{R} 1 / \mathrm{P} 2{ }^{\mathrm{GFP}}\right)$ led to the most pronounced decrease in the percentage of Runx3-expressing TrkC neurons (Fig. 2F,G). Moreover, the remaining $\sim 50 \%$ of TrkC/Runx3 neurons displayed marked reduction in Runx3 intensity (Fig. $2 \mathrm{H}$ ). The critical role of R1 in mediating Runx3 expression was further emphasized by the observation that R1 alone drove Runx3 expression in $>80 \%$ of TrkC neurons (Fig. 2G). Similar results were obtained using BAC-C-delR1 transgenic embryos (Supplemental Fig. S4A,B). Conversely, deletion of $\mathrm{R} 2$ in either BAC transgenic-modified or CRISPR/Cas9modified embryos had almost no effect on Runx3 expression (Fig. 2F,G; Supplemental Fig. S4A,B). Deletion of R3 modestly affected both the percentage of reporter-expressing or Runx3-expressing cells and Runx3 expression intensity (Fig. 2F-H; Supplemental Fig. S4A,B). Of note, the activity of R3 alone at E12.5 was lower than at E11.5, as evidenced by the reduction in percentage and intensity of Runx3 expression in CR $\Delta \mathrm{R} 1,2 / \mathrm{P} 2{ }^{\mathrm{GFP}}$ mutant embryos (Fig. 2). Interestingly, deletion of both R1 and R3 completely abolished reporter or endogenous Runx3 expression in TrkC neurons (Fig. 2F-H), indicating that, unlike at E11.5, R2 does not function alone at E12.5. Thus, both BAC transgenic-modified and CRISPR/Cas9modified embryos showed that, at E12.5, R1 is the dominant RE, R3 exhibits weak activity, and R2 does not confer Runx3 expression in TrkC neurons. Of note, at this developmental stage, Runx3 was detected in wild-type and various $\mathrm{CR} \triangle \mathrm{RE}$ mutants as well as in non-TrkC neurons (Fig. $2 \mathrm{~F})$, as discussed below.

\section{Loss of Runx3 at E12.5 results in depletion of TrkC neurons at later developmental stages}

Cell count of TrkC/GFP neurons in serial sections of various RE-deleted/P2 GFP compound embryos revealed that, in most cases, neurons that had lost Runx3 expression at E12.5 disappeared at E15.5. This conclusion was supported by several lines of evidence: First, at E15.5 and E17.5, in all brachial DRGs, the presence of R1 was sufficient for maintaining a number of TrkC/Runx3 neurons similar to that in wild-type/P2 ${ }^{\text {GFP }}$ control (Fig. 3A,B; Supplemental Figs. S5, S6). This result corresponds with the observation that R1 alone conferred Runx3 expression to most TrkC/GFP neurons even at E12.5. In contrast, in the absence of R1, $40 \%$ of TrkC neurons were lost at E15.5, similar to the percentage of neurons lacking Runx3 at E12.5 (Figs. 2G, 3A). Of note, a fraction of the remaining TrkC/GFP neurons in R1-deleted DRGs did not express Runx3 (Fig. 3A,B; Supplemental Figs. S5, S6), indicating that these cells can survive without Runx3. These Runx3-lacking neurons persisted also at E17.5 (data not shown). Second, at E15.5, in embryos bearing only R2, no Runx3-expressing TrkC/GFP neurons survived (Fig. 3B), as anticipated from the lack of Runx3 expression in these neurons already at E12.5 (Fig. 2G). Last, in the absence of $\mathrm{R} 3, \sim 80 \%$ of TrkC/GFP neurons survived, and most of them expressed Runx3 (Fig. 3A,B), while, in the presence of R3 alone, most TrkC/GFP neurons that retained low levels of Runx3 at E12.5 (Fig. 2G 40\%) survived at E15.5 (Fig. 3A). 


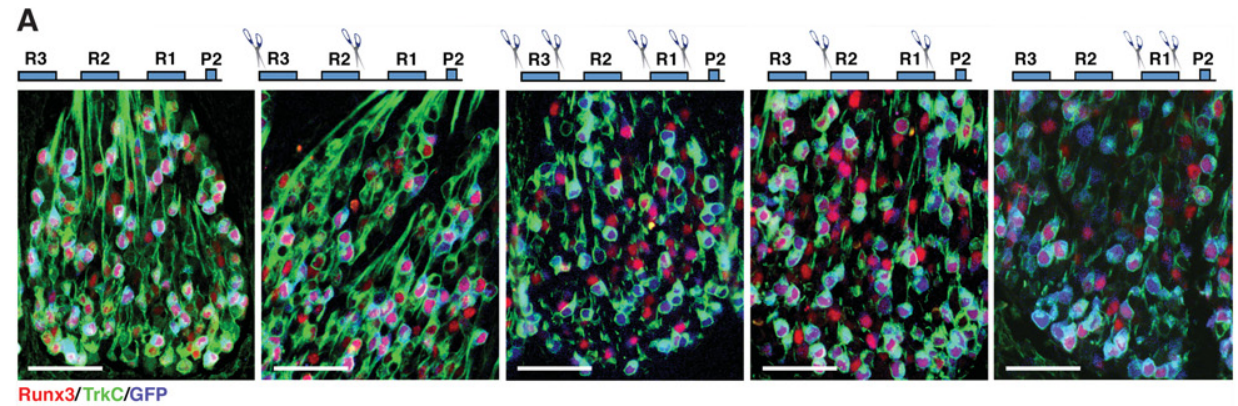

B

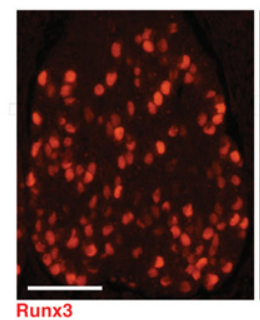

C

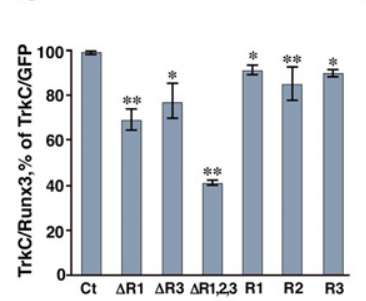

D

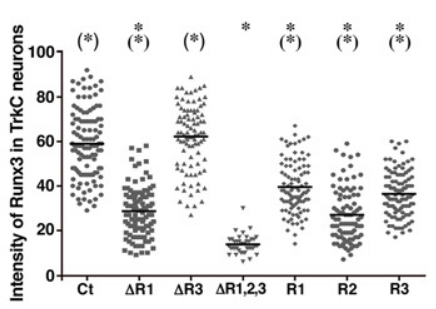

E

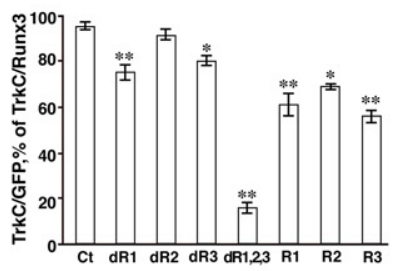

$\mathbf{F}$

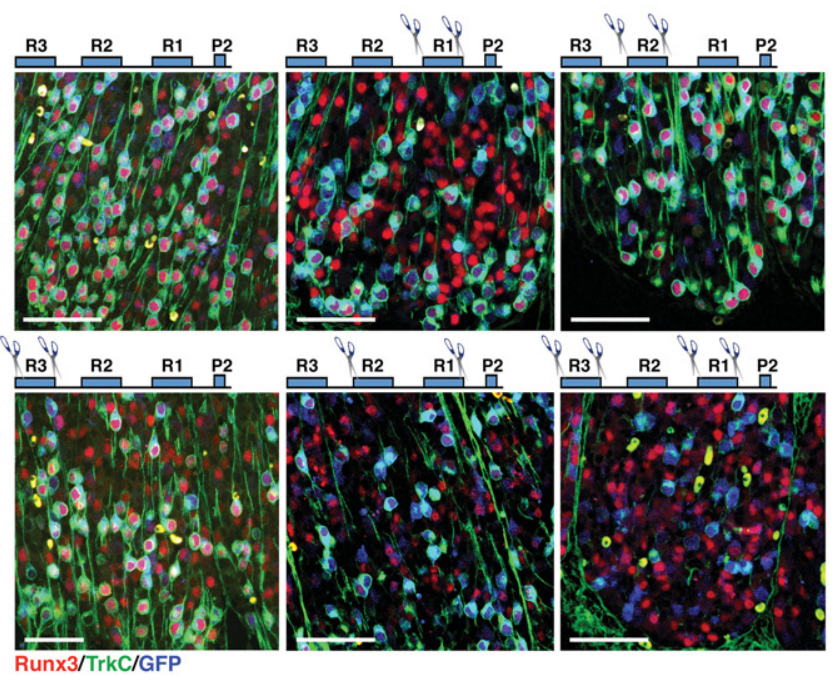

G

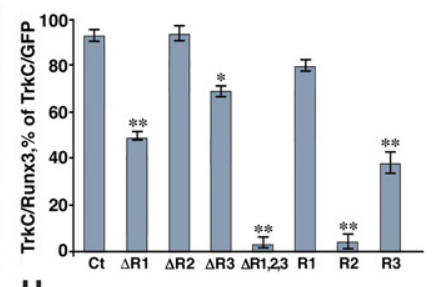

$\mathrm{H}$

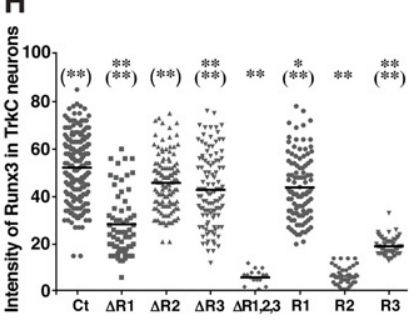

Figure 2. RE activity at E11.5-E12.5. $(A-E)$ At E11.5, the three REs synergize in promoting Runx3 expression. $(A)$ Expression of Runx3, TrkC, and GFP in DRG sections of various CR $\triangle$ RE mutants. (B) Runx3 expression in the same sections as in $A$. $(C)$ The percentage of TrkC/GFP neurons that express Runx3 in RE-deleted embryos. (Ct) Wild-type/P2 ${ }^{\mathrm{GFP}}$; $(\Delta \mathrm{R} 1) \mathrm{CR} \Delta \mathrm{R} 1 / \mathrm{P} 2^{\mathrm{GFP}}$; $(\Delta \mathrm{R} 3) \mathrm{CR} \Delta \mathrm{R} 3 / \mathrm{P} 2^{\mathrm{GFP}}$; $(\Delta \mathrm{R} 1,2,3) \mathrm{CR} \Delta \mathrm{R} 1,2,3 / \mathrm{P} 2 \mathrm{GFP} . \mathrm{R} 1, \mathrm{R} 2$, and $\mathrm{R} 3$ represent the remaining single $\mathrm{RE}$ following removal of the two other REs. $(*) P<0.005$; $\left.{ }^{* *}\right) P<0.0001$. (D) Runx3 expression intensity. $\left({ }^{*}\right) P<0.0001$ compared with $\mathrm{Ct}$; $\left.\left[{ }^{*}\right)\right] P<0.0001$ compared with $\Delta \mathrm{R} 1,2,3$. $(E)$ Percentage of TrkC/Runx3 neurons expressing GFP from BAC-C-GFP $(\mathrm{Ct})$ and its RE-deleted $(\mathrm{d})$ variants. $\left({ }^{*}\right) P<0.005$; $\left({ }^{* *}\right) P<0.0001$. $(F-H)$ Differential RE activity at E12.5. (F) Runx3, TrkC, and GFP expression at E12.5 in various CR $\triangle$ RE genotypes. (G) The percentage of Runx3/TrkC/ GFP neurons out of TrkC/GFP neurons. $\left.\left(^{*}\right) P<0.005 ;{ }^{* *}\right) P<0.0001$. $(H)$ Intensity of endogenous Runx3 expression in TrkC neurons at E12.5. (*) $P<0.0002$; $\left(^{* *}\right) P<0.0001$ compared with $\mathrm{Ct}$; $[(*)] P<0.0002 ;[(* *)] P<0.0001$ compared with $\Delta \mathrm{R} 1,2,3$. Bar, $50 \mu \mathrm{m}$. 

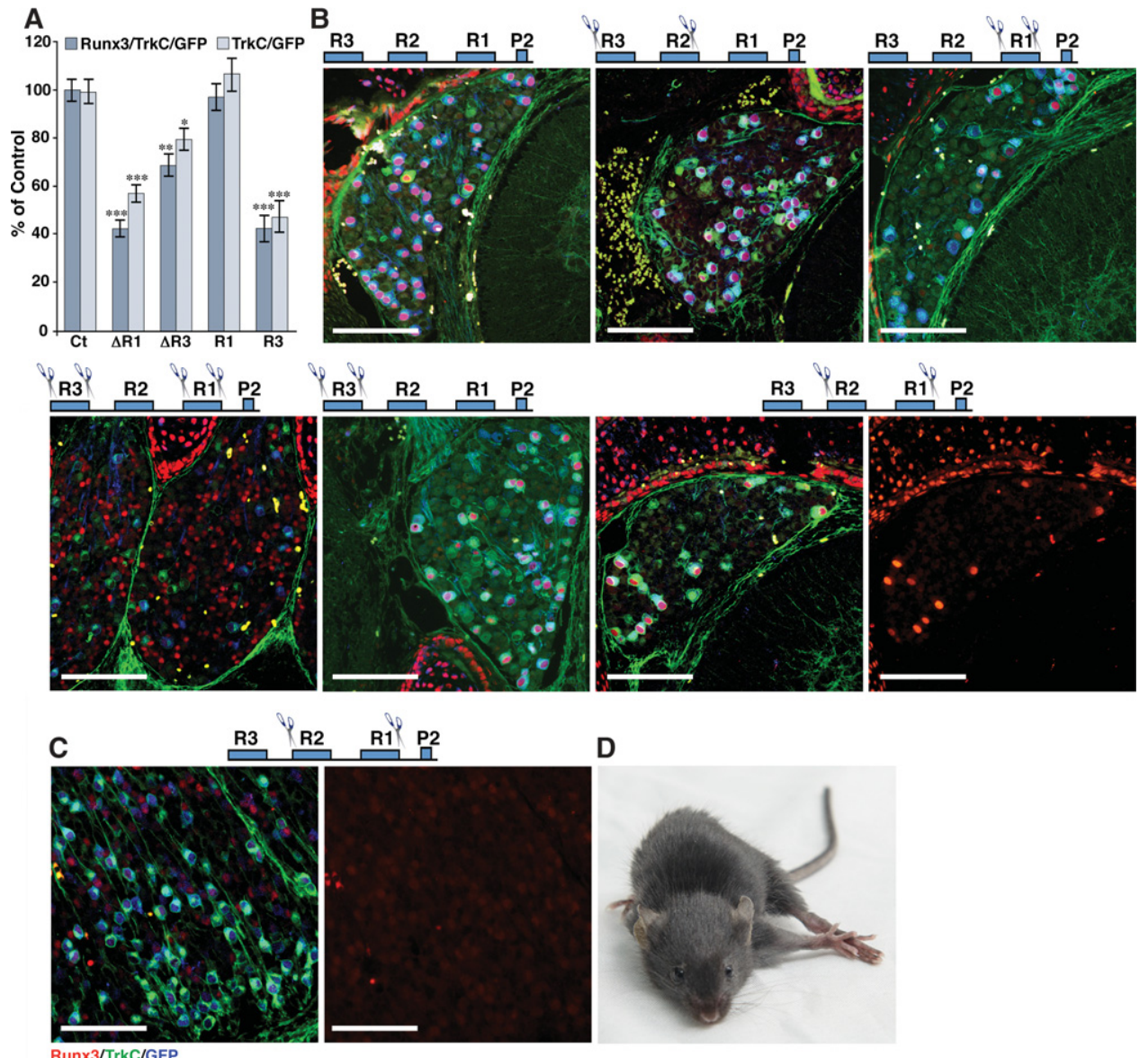

Figure 3. RE deletion causes a differential loss of TrkC/Runx3 neurons at E15.5. (A) The percentage of Runx3/TrkC/GFP (dark gray) or total TrkC/GFP neurons (light gray) surviving in various $\triangle \mathrm{RE}$ embryos relative to the corresponding neuron numbers in Ct ganglia. The percentage was determined in serial sections of C6-T1 ganglia (Supplemental Fig. S5). $\left({ }^{*}\right) P<0.05 ;\left(^{* *}\right) P<0.001 ;\left({ }^{* *}\right) P<0.0001$. $(B)$ Expression of Runx3, TrkC, and GFP at E15.5 in C7-C8 ganglia. The last panel at the right in the bottom row shows Runx3 expression in $\triangle \mathrm{R} 1,2$ in the same DRG section as the one adjacent at its left. (C) As detailed in $B$ for $\Delta \mathrm{R} 1,2$ at E12.5 (left panel) and Runx3 expression in the same section (right panel). (D) $\Delta \mathrm{R} 1,3$ mice encounter severe congenital ataxia. Picture of a 3-wk-old $\Delta \mathrm{R} 1,3$ mouse (see Supplemental Movie S1). Bar, $50 \mu \mathrm{m}$.

Remarkably, at E15.5, the intensity of Runx3 in neurons bearing only R3 was similar to that in the control and much higher than at E12.5 (Fig. 3B,C). This finding indicates that R3 efficacy increased with embryonal age. Interestingly, RE mutants exhibited a similar pattern of Runx3 loss in the trigeminal ganglion (TG). Like in DRGs, deletion of either all three REs or only the duo $\mathrm{R} 1$ and R3 led to the complete loss of Runx3 expression in TG neurons, whereas removal of R1 alone resulted in the loss of Runx3 in many, but not all, TG TrkC neurons. However, unlike in DRGs, a substantial number of TG TrkC neurons still survived in the absence of Runx3 (Supplemental Fig. S7).

\section{Mice lacking both $R 1$ and $R 3$ develop severe} $\operatorname{limb}$ ataxia

As shown before, mice lacking the entire 25-kb genomic region harboring the three REs developed severe limb ataxia. Importantly, a precise deletion of R1 and R3 had a similar effect despite the retention of $\mathrm{R} 2$ and flanking sequence (Fig. 3D; Supplemental Movie S1). Of note, although Runx3 was expressed at E11.5 in DRGs lacking both R1 and R3 (Fig. 2A-E), this early R2-mediated Runx3 expression did not rescue TrkC neurons at later stages. In fact, just $1 \mathrm{~d}$ later (E12.5), the entire TrkC neuron population nearly disappeared in mice bearing $\mathrm{R} 2$ alone (Fig. 2F-H). The early loss of TrkC neurons in the embryo is compatible with the severe ataxia observed in these newborn mice. Conversely, the presence of R3 alone (which, at E12.5, mediated low levels of Runx3/TrkC expression) was sufficient for rescuing a subpopulation of TrkC neurons. Moreover, these R3-only (CR $\Delta \mathrm{R} 1,2)$ mice did not develop ataxia. The increase in R3 activity during later embryonic development may explain the seemingly normal gait of R3-only mutant mice (Fig. 3B,C). These results demonstrate that loss of $\mathrm{R} 1+\mathrm{R} 3$ per se is sufficient for ataxia development. 
Loss of the R1-dependent TrkC neuronal subpopulation is associated with reduced central afferents and defects in mouse locomotor activity

To further investigate the function of the three REs, we immunostained the spinal cords of various CR $\triangle \mathrm{RE} / \mathrm{P} 2 \mathrm{GFP}$ mouse mutants for the $\mathrm{Ca}^{++}$-binding protein parvalbumin (Pvalb), which is expressed in most TrkC/Runx3 neurons starting at E14.5. This enabled us to monitor spinal cord proprioceptor-central afferents at late embryonic stages. Deletion of all three REs resulted in the complete loss of central afferents corresponding to the aforementioned early loss of TrkC neurons in CR $\Delta \mathrm{R} 1,2,3$ mice. A solitary R3 deletion caused only a minor effect, whereas $\mathrm{R} 1$ deletion led to a substantial reduction in the number of central afferents. Deletion of both R1 and R2, retaining only R3, exacerbated the loss of ventral afferents as compared with R1 deletion (Fig. 4A). This result reflects the relatively small size of the R3-dependent subpopulation and the late onset of Runx 3 expression in this population (Fig. 3B,C), which is essential for afferent extension (Lallemend et al. 2012). Nevertheless, as noted before, this small number of afferents sufficed to support an outwardly normal gait.

To associate neuronal loss with functional consequences, we subjected the mice to several basic behavioral tests.
The home cage locomotion test, which quantifies voluntary spontaneous activity in the home cage, revealed almost no difference between CR $\Delta \mathrm{R} 1 / \mathrm{P} 2^{\mathrm{GFP}}$ and $\mathrm{CR} \Delta \mathrm{R} 3 /$ $\mathrm{P} 2{ }^{\mathrm{GFP}}$ mice as compared with their respective wild-type/ $\mathrm{P} 2{ }^{\mathrm{GFP}}$ controls. On the other hand, $\mathrm{CR} \Delta \mathrm{R} 1,2 / \mathrm{P} 2{ }^{\mathrm{GFP}}$ mice displayed significantly lower mean hourly activity (Fig. 4B). Performance in the horizontal beam-walking test required precise foot placing and assessed forced or challenged motor balance and coordination (Luong et al. 2011). In this test, both $\mathrm{CR} \Delta \mathrm{R} 1,2 / \mathrm{P} 2^{\mathrm{GFP}}$ and $\mathrm{CR} \Delta \mathrm{R} 1 /$ P2 ${ }^{\mathrm{GFP}}$ mice displayed a significantly higher rate of slippage from the beam relative to control wild-type/P2 ${ }^{\mathrm{GFP}}$ mice. Effect size analyses revealed that the absence of both R1 and R2 was associated with more frequent foot slippage compared with the loss of only R1 (Fig. 4C). The time lapse required for beam crossing and the number of steps on the beam also tended to be higher in the $\mathrm{CR} \Delta \mathrm{R} 1,2 / \mathrm{P} 2^{\mathrm{GFP}}$ group (data not shown). The poorer performance of $\mathrm{CR} \Delta \mathrm{R} 1 / \mathrm{P} 2^{\mathrm{GFP}}$ and $\mathrm{CR} \Delta \mathrm{R} 1,2 / \mathrm{P} 2^{\mathrm{GFP}}$ mice relative to $\mathrm{CR} \Delta \mathrm{R} 3 / \mathrm{P} 2^{\mathrm{GFP}}$ mice in the beam-walking test indicates that retention of the R1-dependent population is mandatory for foot-placing accuracy. The increased effect of $\Delta \mathrm{R} 1,2$ compared with $\Delta \mathrm{R} 1$ in the behavioral tests uncovered a functional role for $\mathrm{R} 2$, the physiological ramification of which is described below.

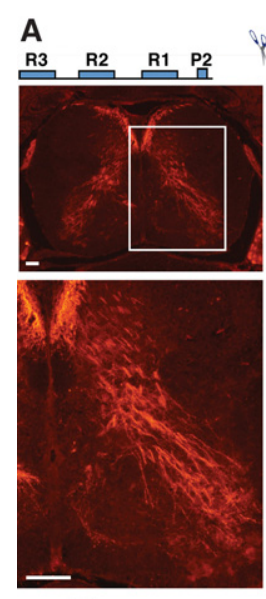

B

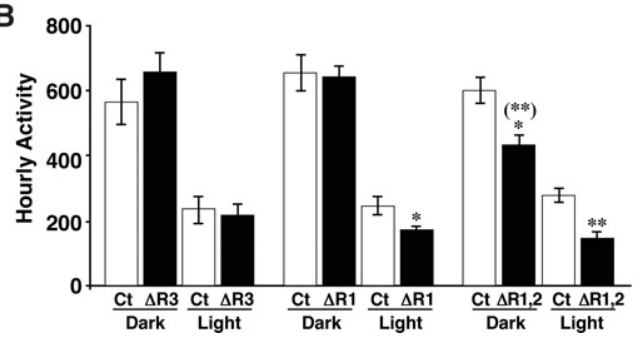

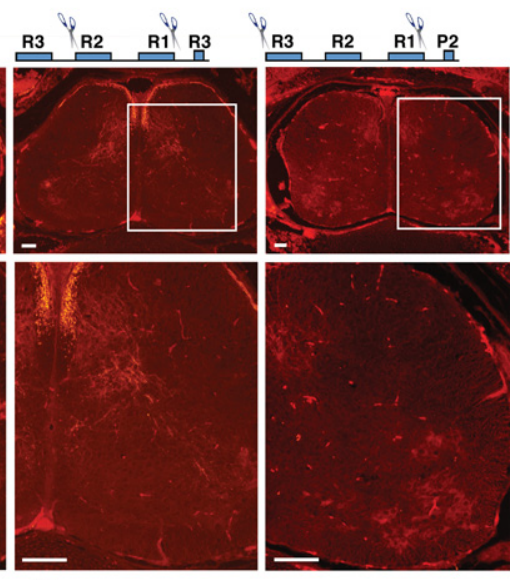

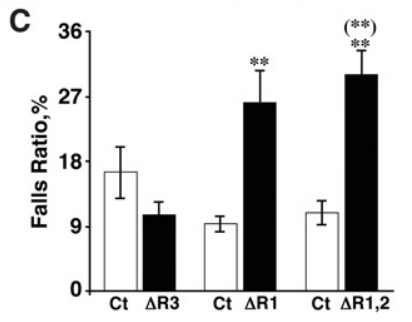

Figure 4. Projection of central afferents and functional analysis of RE-dependent TrkC/Runx3 subpopulations. $(A)$ Central afferents of E17.5 C7 DRGs in sections of various RE-deleted embryos visualized by Pvalb staining. The bottom panels depict the corresponding enlarged insets. $(B)$ Home cage locomotion: mean hourly activity assay during dark/active and light/dormant phases. $\Delta \mathrm{R} 3(n=9), \Delta \mathrm{R} 1(n=$ 25), $\Delta \mathrm{R} 1,2(n=16)$ male mice were compared with age-matched wild-type/P2 ${ }^{\mathrm{GFP}}(\mathrm{Ct})$ mice $(n=10, n=12$, and $n=27$, respectively). (*) $P<$ $\left.0.05 ;{ }^{* *}\right) P<0.01$ compared with $\mathrm{Ct}$, Student $t$-test. $[(* *)] P<0.01 \Delta \mathrm{R} 1,2<\Delta \mathrm{R} 3$ and $\Delta \mathrm{R} 1$, ANOVA followed by Scheffe post-hoc comparisons. (C) Beam-walking assay. The same mice as in $B$ were examined. Falling ratio is shown; Mann-Whitney comparisons indicate $\Delta \mathrm{R} 1>\mathrm{Ct}(P=$ $0.003)$ and $\Delta \mathrm{R} 1,2>\mathrm{Ct}(P=0.000) .\left(^{* *}\right) P<0.01$. There is a difference in the effect sizes $(r)$ between $\Delta \mathrm{R} 1$ versus $\mathrm{Ct}(0.462)$ and $\Delta \mathrm{R} 1 \mathrm{R} 2$ versus $\mathrm{Ct}(0.700) ; \Delta \mathrm{R} 1$ is considered "medium" $(0.3><0.5)$, while $\Delta \mathrm{R} 1 \mathrm{R} 2$ is considered "large" $(>0.5)$. Kruskal-Wallis followed by Dunn's corrected pair-wise comparison. $[(* *)] P=0.003 \Delta \mathrm{R} 1,2>\Delta \mathrm{R} 3$. Bar, $50 \mu \mathrm{m}$. 
Appel et al.

Differential activity of R1-R3 determines Runx3 expression in distinct subpopulations of DRG TrkC neurons

Our observation that loss of both R1 and R3, but not of each of them alone, resulted in severe ataxia indicates that the function of these two REs in TrkC neurons is additive. To further evaluate the interrelationship between the three REs in different ganglia, we determined the number of surviving TrkC/Runx3 neurons in C5-T1 DRGs of CR $\triangle$ RE/P2 ${ }^{\text {GFP }}$ mutants and wild-type/P2 GFP control mice. In DRGs at spinal level C5, deletion of R1 or R3 had little or no effect, respectively (Fig. 5A; Supplemental Figs. S5, S6), suggesting that the activity of R1 and $\mathrm{R} 3$ in this ganglion is redundant. Further analysis revealed that R1 on its own mediated Runx3 expression in all TrkC/GFP neurons (Supplemental Figs. S5, S6), whereas R3 conferred expression in $~ 55 \%$ of the neurons, implying that the apparent redundancy between them is limited to that population (Fig. 5A,A2). Another R1-dependent subpopulation, comprising $15 \%$ of $\mathrm{C} 5 \mathrm{TrkC} / \mathrm{Runx} 3$ neurons, consisted of neurons that lost Runx3 upon R1 deletion
(Fig. 5A,A1). The remaining 30\% of C5 TrkC/Runx3 neurons endured either $\mathrm{R} 1$ or $\mathrm{R} 3$ deletion due to redundant activity of R1 and R2 + R3 (Fig. 5A,A3). This last conclusion is based on the observation that while $\mathrm{R} 3$ alone drove Runx3 expression in $55 \%$ of TrkC/Runx3 neurons, the combination of R2 + R3 conferred expression in $85 \%$ of them. The data indicate that $\mathrm{R} 2$, which is not active alone, supports R3 activity in the A3 population (Fig. 5A,A3). In summary, counting all TrkC/Runx3 neurons in the C5 ganglion of the various $\mathrm{CR} \triangle \mathrm{RE} / \mathrm{P} 2^{\mathrm{GFP}}$ mutant mice defined three neuronal populations: A1, an R1-dependent population comprising $15 \%$ of C5 TrkC/Runx 3 neurons; $\mathrm{A} 2$, an $\mathrm{R} 1 / \mathrm{R} 3$-dependent population comprising $55 \%$ of C5 TrkC/Runx3 neurons; and A3, an R1/R2 and R3-dependent population comprising $30 \%$ of these neurons.

In C6-C7 ganglia, unlike in C5, R1 deletion caused a pronounced $(\sim 50 \%)$ loss of TrkC/Runx3 neurons (Fig. 5B; Supplemental Figs. S5, S6). Thus, 50\% of TrkC neurons in these ganglia are R1-dependent (Fig. 5B,B1). R3 deletion showed that an additional $30 \%$ of $\mathrm{C} 6-\mathrm{C} 7$ neurons are R3-dependent, as its deletion eliminated $30 \%$ of TrkC/Runx3 neurons, and, vice versa, R3 alone mediated
A

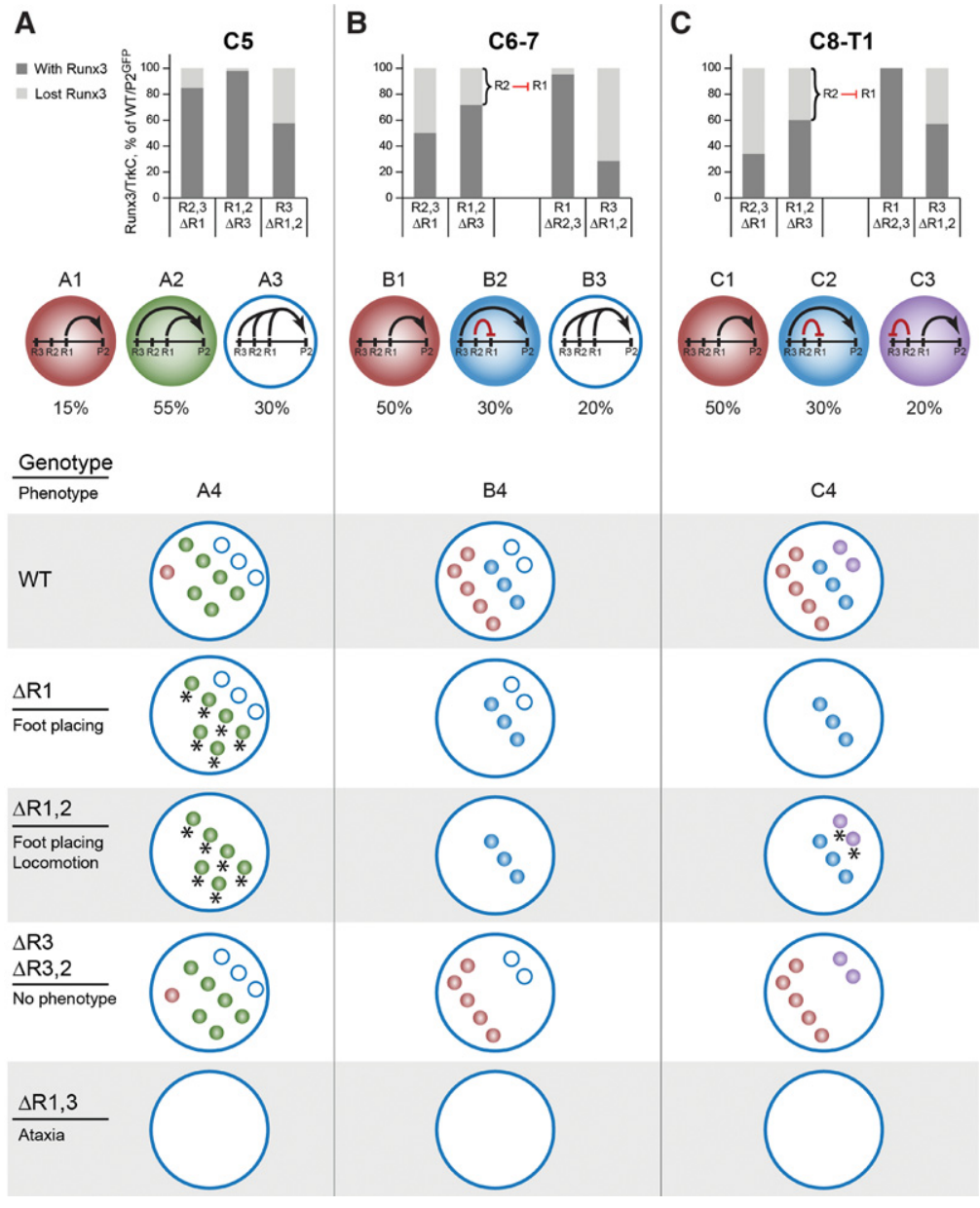
subpopulation is similar to B2. (C3) The R1-dependent subpopulation, in which R2 represses R3. (C4) Similar to A4 and B4 for C8 and T1 ganglia.
Figure 5. Distinct RE combinations mediate Runx3 expression in TrkC neuronal subpopulations. $(A)$ Percentage of Runx3-expressing TrkC/GFP neurons that persisted (gray) or were lost (off-white) at E15.5 in ganglion C5 in the various RE-deleted embryos compared with the wild-type/P2 ${ }^{\mathrm{GFP}}$ control (based on Supplemental Figs. S5, S6). (A1) A scheme illustrating the R1-dependent subpopulation. (A2) A scheme illustrating the R1/R3-dependent subpopulation. (A3) Runx3 is regulated by either $\mathrm{R} 1$ or $\mathrm{R} 2$ in cooperation with $\mathrm{R} 3$, giving rise to the $\mathrm{R} 1 / \mathrm{R} 2$ and R3-dependent subpopulation. The percentage of each subpopulation out of the total wild-type/ $\mathrm{P} 2{ }^{\mathrm{GFP}}$ neurons is indicated below. (A4) Schematic representation of the relative abundance of the surviving subpopulations. The number of color-coded circles corresponds to the A1, A2, and A3 subpopulations. The green-coded subpopulation marked by asterisks in $\Delta \mathrm{R} 1$ and $\Delta \mathrm{R} 1,2$ differs from the similar subpopulation in wild-type or $\Delta \mathrm{R} 3, \Delta \mathrm{R} 2,3$ in their late Runx3 expression onset. The genotype and phenotype of each mutant is shown at the left. (B) Analysis was conducted as detailed in $A$ for the C6-C7 ganglia. The bracket at the $\mathrm{R} 1,2$ histogram indicates the R3-dependent subpopulation that disappears in $\Delta \mathrm{R} 3$ mutants due to repression of $\mathrm{R} 1$ by $\mathrm{R} 2$. (B1) The R1-dependent Runx3/TrkC subpopulation is similar to A1. (B2) The R3-dependent Runx3/TrkC subpopulation. The scheme illustrates a situation in which Runx3 is regulated by $\mathrm{R} 3$, while $\mathrm{R} 1$ is repressed by R2. (B3) The R1/R2 and R3 subpopulation, similar to A3. (B4) As in A4 for the C6 or C7 ganglion. (C) Analysis was conducted as detailed in $A$ for the C8-T1 ganglia. The bracket at the $\mathrm{R} 1,2$ histogram indicates the R3-dependent subpopulation as in B. (C1) The R1-dependent subpopulation similar to A1 and B1. (C2) The R3-dependent R2 represses R3. (C4) Similar to A4 and B4 for C8 
Runx3 expression in $~ 30 \%$ of TrkC neurons (Fig. 5B,B2). In the remaining $20 \%$ of TrkC neurons, Runx 3 expression was redundantly regulated by $\mathrm{R} 1$ plus $\mathrm{R} 2$ and $\mathrm{R} 3$ (Fig. 5B, $\mathrm{B} 3)$. This $\mathrm{B} 3$ population in $\mathrm{C} 6-\mathrm{C} 7$ ganglia is similar to the A3 subpopulation in the C5 ganglion. We also noted that the R1 plus $\mathrm{R} 2(\Delta \mathrm{R} 3)$ population, comprising $70 \%$ of TrkC/Runx3 neurons, was significantly smaller compared with the R1-only $(\Delta \mathrm{R} 2,3)$ population, which comprised $\sim 100 \%$ of TrkC/Runx3 neurons (Fig. 5B). This result indicates that $\mathrm{R} 2$ represses $\mathrm{R} 1$ activity in $30 \%$ of TrkC/Runx3 neurons (Fig. 5B,B2). Accordingly, the R1-repressed population corresponds to the R3-dependent $(\Delta \mathrm{R} 1,2)$ population.

In the C8-T1 ganglia, a similar pattern of R1 and R3 segregation generated three subpopulations, of which $\mathrm{C} 1$ is analogous to $\mathrm{A} 1$ and $\mathrm{B} 1$, and $\mathrm{C} 2$ is analogous to $\mathrm{B} 2$ (Fig. $5 \mathrm{C}, \mathrm{C} 1, \mathrm{C} 2$ ). The third C8-T1 subpopulation (Fig. 5C,C3) was inferred by combined R1 activity and R2-mediated repression of R3. This interpretation was borne out of the observation that, in the R3-alone $(\Delta \mathrm{R} 1,2)$ subpopulation, Runx3 was expressed in $\sim 50 \%$ of the neurons, whereas the presence of $\mathrm{R} 3$ and $\mathrm{R} 2$ (i.e., Fig. $5 \mathrm{C}, \Delta \mathrm{R} 1$ or $\mathrm{R} 2,3$ ) reduced the number of Runx3-expressing TrkC neurons to $30 \%$. Taken together, these results reveal that, in the C5-T1 DRGs, TrkC neurons segregate into five distinct subpopulations according to their dependence on various combinations of RE-mediated Runx3 expression: (1) R1dependent (A1, B1, and C1), (2) R3-dependent (B2 and C2), (3) R1/R3-dependent (A2), (4) R1/R2 and R3-dependent (A3 and B3), and (5) R1-dependent with R3 repressed by R2 (C3). The differences in abundance of these subpopulations along consecutive brachial DRGs are likely to reflect subtle changes in the proprioceptive properties of their respective target muscles.

Importantly, these neuronal subpopulations differ functionally, as demonstrated by the behavioral tests. Specifically, the precision of foot placing in the beam test singled out the R1-dependent subpopulations (A1, B1, C1, and $\mathrm{C} 3$ ), as the loss of these subpopulations increased foot slippage (Figs. 4C, 5A-C [ $\Delta \mathrm{R} 1$ in A4,B4,C4]). Conversely, the home cage locomotion scores of mutants lacking these R1-dependent populations were similar to those of wildtype mice, suggesting that they are less important for gross motor function. However, upon double deletion of $\mathrm{R} 1$ and $\mathrm{R} 2\left(\Delta \mathrm{R} 1,2 / \mathrm{P} 2^{\mathrm{GFP}}\right)$, which resulted in loss of the main R1-dependent subpopulations A1, B1, and C1 as well as the A3 and B3 subpopulations, we observed a reduction in home cage hourly activity and exacerbation of foot slippage in comparison with $\Delta \mathrm{R} 1$. Thus, the further removal of the $\mathrm{A} 3$ and $\mathrm{B} 3$ subpopulations, retaining only the R3-dependent subpopulations (B2 and C2), impaired both mouse locomotion and coordination. Moreover, deletion of these R3-dependent subpopulations (Fig. 5B, $\mathrm{C}, \Delta \mathrm{R} 3$ and $\Delta \mathrm{R} 3,2$ in $\mathrm{B} 4, \mathrm{C} 4)$ was not associated with any behavioral defect, supporting the conclusion that R3dependent populations are not essential for gross locomotion or precise foot placing. Thus, an intricate combinatorial interplay among the three REs drives Runx3 expression in distinct DRG neuronal subpopulations that play specific functional roles.
$R 1$ represses $R 2$-driven Runx3 expression in Trk $A$ neurons

In addition to the high expression levels in wild-type TrkC neurons, we also detected lower Runx3 levels in nonTrkC neurons of E11.5 and E12.5 embryos (Fig. 2A,F). Interestingly, this early Runx3 expression was confined to $\sim 40 \%-50 \%$ of TrkA neurons (Fig. 6 A,C). Because the percentage of Runx3-expressing TrkA neurons in mutant embryos lacking the three REs was similar to that in wildtype/P2 ${ }^{\mathrm{GFP}}$ mice, we deduced that early Runx3 expression in TrkA neurons was driven solely by P2. Importantly, P2 activity in TrkA neurons disappeared at a later embryonic stage, as demonstrated by the lack of Runx3 in either $\mathrm{CR} \triangle \mathrm{R} 1,2,3 / \mathrm{P} 2^{\mathrm{GFP}}$ or wild-type E14.5 TrkA neurons (Fig. $6 \mathrm{~B}, \mathrm{C})$. Of note, upon deletion of $\mathrm{R} 1$ alone (CR $\Delta \mathrm{R} 1 /$ $\mathrm{P} 2^{\mathrm{GFP}}$ ), Runx3 was readily detected in most E14.5 TrkA neurons (Fig. 6B,C). Thus, R1 simultaneously promotes Runx3 expression in TrkC neurons and represses it in TrkA neurons. This conclusion was supported by a similar result obtained using BAC transgenics (Fig. 6D,E). Importantly, in contrast to its inability to drive Runx3 expression in TrkC neurons, $\mathrm{R} 2$ alone (CR $\Delta \mathrm{R} 1,3 / \mathrm{P} 2{ }^{\mathrm{GFP}}$ ) conferred Runx3 expression in TrkA neurons (Fig. 6B,C). Moreover, in BAC transgenics, reporter expression in TrkA neurons was driven only by constructs bearing R2 and lacking R1 but not in mutants lacking R2. Collectively, the complementary results of in vivo CR $\triangle \mathrm{RE} / \mathrm{P} 2^{\mathrm{GFP}}$ mutants and BAC transgenics demonstrate that R2 positively regulates Runx3 expression in TrkA neurons. R3 can cooperate with $\mathrm{R} 2$, as a higher percentage of TrkA neurons expressed Runx3 when both R2 and R3 were present (CR $\left.\Delta \mathrm{R} 1 / \mathrm{P} 2^{\mathrm{GFP}}\right)$, as compared with $\mathrm{R} 2$ alone (Fig. 6C). This occurrence was particularly pronounced in E14.5 mutant embryos. In the context of the spatiotemporal regulation, the data demonstrate that Runx3 expression specificity in TrkC neurons is determined by not only R1-enhancive activity but also R1-mediated Runx3 repression in TrkA neurons (Fig. 6F).

Runx3 is not expressed in wild-type TrkB neurons (Supplemental Fig. S8A,C). However, deletion of R2 from BACC (BAC-C-delR2) revealed an early stage (E11.5) BAC-mediated GFP expression in a small number of TrkB neurons that, at E12.5-E14.5, comprised nearly $80 \%$ of these neurons (Supplemental Fig. S8B,D). This observation implies that, in the context of BAC transgenics, R2 mediates Runx3 repression in TrkB neurons and that $\mathrm{R} 2$ activity may be essential for TrkB neuron survival. However, analysis of E12.5 CR $\Delta \mathrm{R} 2 / \mathrm{P} 2^{\mathrm{GFP}}$ or homozygous $\Delta \mathrm{R} 2 / \Delta \mathrm{R} 2$ embryos revealed only a small population of Runx3 expressing TrkB neurons (Supplemental Fig. S8E). This small population was absent at E17.5, with a minute decline in the number of TrkB neurons in $\Delta \mathrm{R} 2 / \Delta \mathrm{R} 2$ mice compared with control (data not shown). Thus, excision of R2 from BAC-C led to reporter-GFP expression in $\sim 80 \%$ of TrkB neurons, while its deletion in vivo $(\Delta \mathrm{R} 2 / \Delta \mathrm{R} 2)$ produced only a small number of TrkB/Runx3 neurons, indicating that $\mathrm{R} 2$ alone could not repress Runx 3 expression in these cells in-vivo. Together, these results suggest that Runx3 protein levels in TrkB neurons may be regulated at the 


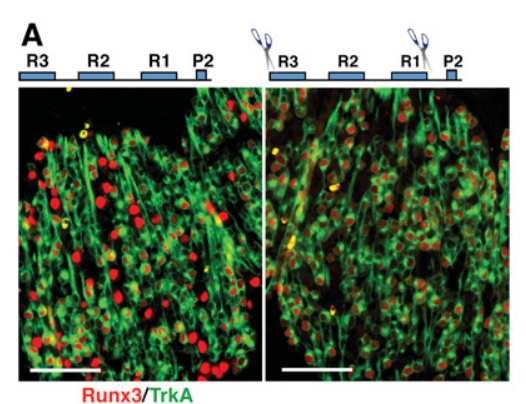

Runx3/TrkA

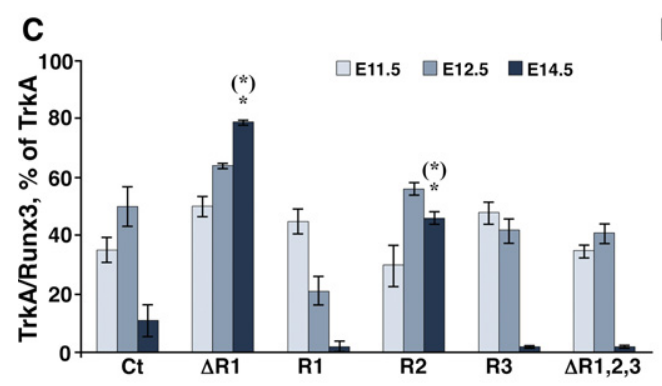

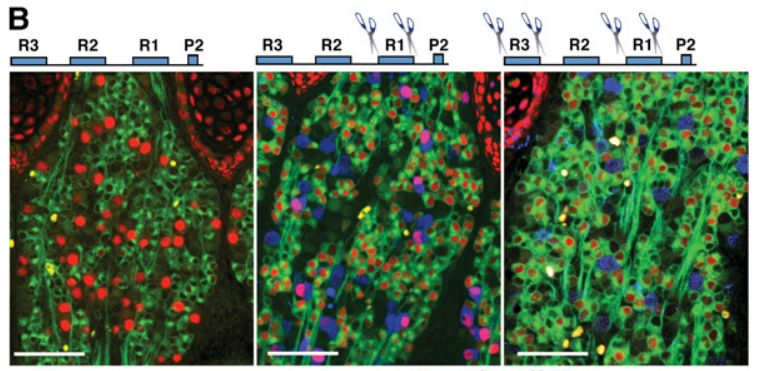

Runx3/TrkA/GFP
D

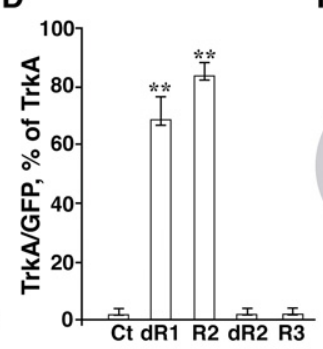

$\mathbf{F}$

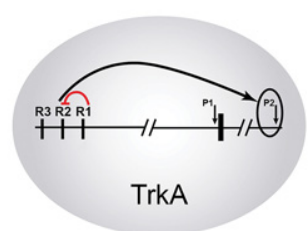

E
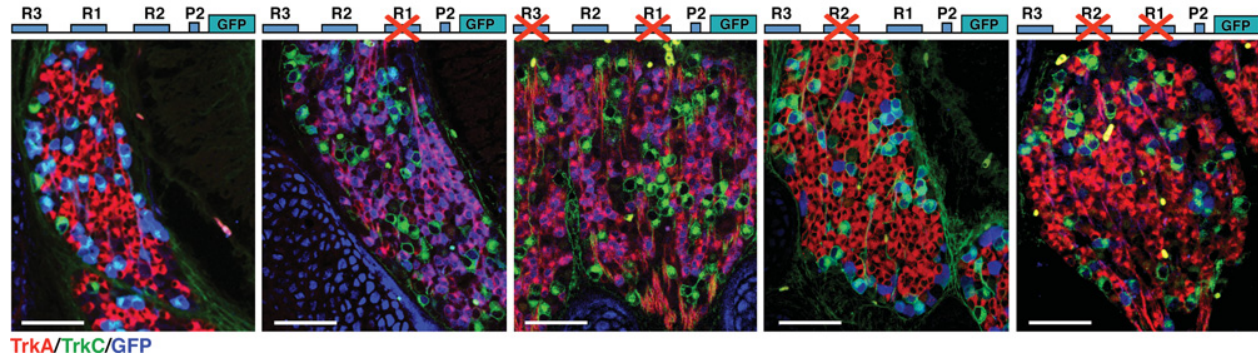

Figure 6. R1 antagonizes $\mathrm{R} 2$ function in TrkA neurons. (A) Runx3 expression in E12.5 TrkA neurons of wild-type/P2 ${ }^{\mathrm{GFP}}$ and $\Delta \mathrm{R} 1,2,3 /$ P2 ${ }^{\text {GFP }}$ mice. $(B)$ Runx3, TrkA, and GFP expression at E14.5 in the indicated embryos. GFP marks TrkC neurons. $(C)$ The percentage of TrkA neurons expressing Runx3 in RE-deleted embryos at E11.5 (light), E12.5 (medium), and E14.5 (dark). (*) $P<0.001$ compared with $\mathrm{Ct}_{;}[(*)] P<0.001$ compared with $\triangle \mathrm{R} 1,2,3$. (D) The percentage of TrkA neurons expressing GFP in BAC transgenics at E14.5. $(E)$ Expression of TrkA, TrkC, and GFP in BAC transgenics at E14.5. (F) Schematic representation of Runx3 silencing in TrkA neurons by R1-mediated repression of R2 activity. Bar, $50 \mu \mathrm{m}$.

translation/degradation level and that the absence of Runx3 in wild-type TrkB neurons may be controlled by mechanisms yet to be elucidated. Alternatively, BAC-C could lack an additional silencing element that is present in the genome, leading to the difference between the two model systems.

\section{$B R N F$ TF-binding sites regulate $R 1$ and $R 3$ activity}

We next sought to identify upstream TFs that regulate Runx3 RE activity. The amount of TrkC neurons that can be isolated from DRGs is insufficient for TF-specific ChIP-seq (chromatin immunoprecipitation [ChIP] combined with high-throughput sequencing) analyses. Therefore, we conducted RNA sequencing (RNA-seq) using RNA isolated from purified TrkC/GFP neurons of wildtype/P2 ${ }^{\mathrm{GFP}}$ and Runx3-deficient homozygous $\mathrm{P} 2{ }^{\mathrm{GFP} / \mathrm{GFP}}$ E11.5 embryos. The major aim of this gene expression analysis was to identify TFs that are refractory to loss of Runx3 and therefore could be considered potential regulators of
Runx3 RE activity. As seen in Supplemental Table S6, several TFs were highly expressed by these neurons, most of which were not affected by the loss of Runx3. Among these TFs, the POU homeodomain Brn3a was implicated previously in Runx3 regulation (Dykes et al. 2010). A conserved Brn3a-binding site located $94 \mathrm{~kb}$ upstream of Runx3 (marked as BrnT in Fig. 7A) was shown previously to bind Brn3a (Dykes et al. 2010). Interestingly, this -94-kb genomic region colocalized with R3, implying that Brn3a is one of the TFs regulating R3 activity. Sequence alignment analysis of the R3 genomic region for conserved TF-binding sites identified both BrnT and an additional Brn3a-binding site (marked BRNF-R3). To directly demonstrate the in vivo participation of Brn3a in the regulation of Runx3 in TrkC neurons, we mutated either the BrnT site or the two BRNF-R3-binding motifs in BAC-C. Both the BrnT and BRNF-R3 mutations caused a similar effect, mirroring the deletion of an entire R3 (Fig. 7B,C). These results support the notion that Brn3a is an important upstream regulator of R3-dependent Runx3 expression in TrkC neurons. 
Downloaded from genesdev.cshlp.org on April 26, 2023 - Published by Cold Spring Harbor Laboratory Press

Driving TrkC neuron-specific Runx3 expression

A

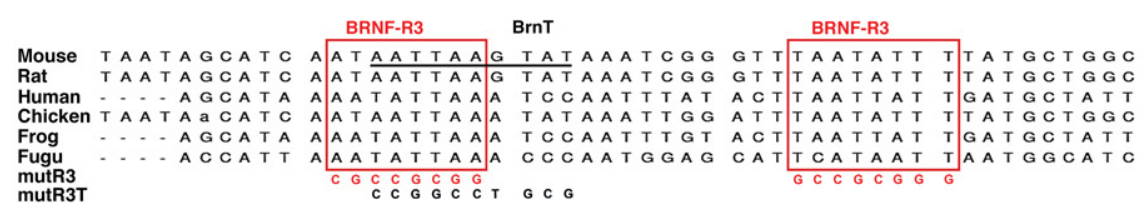

B

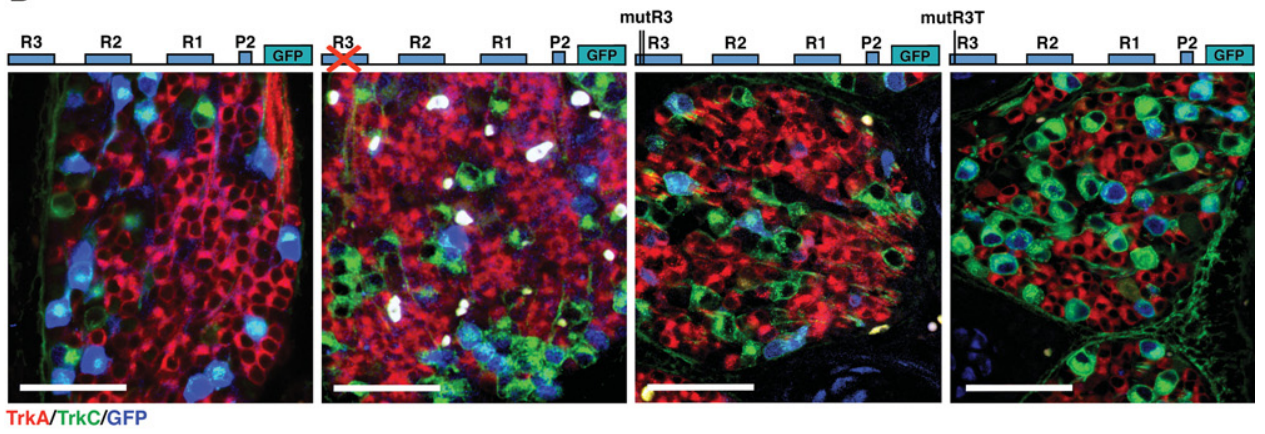

C

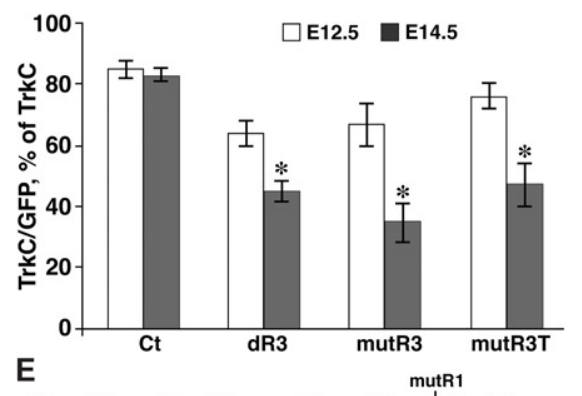

E

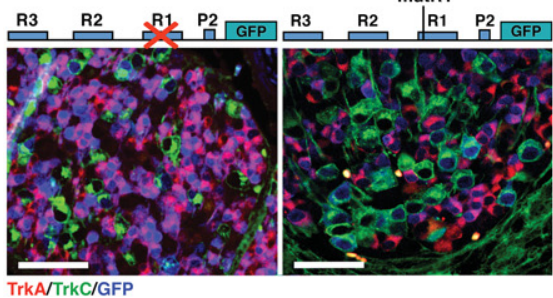

$\mathbf{F}$
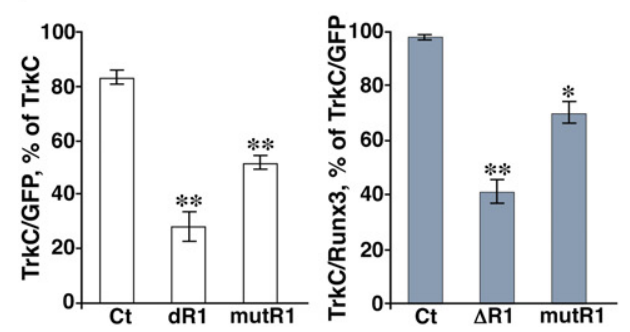

D

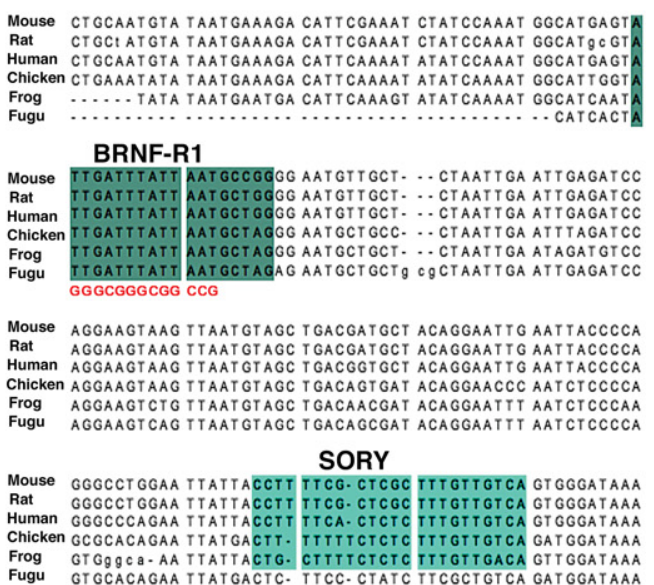

G

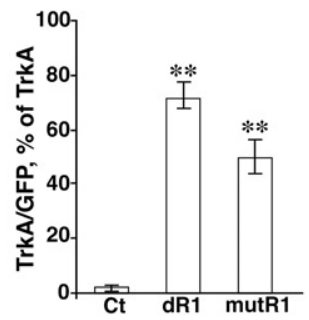

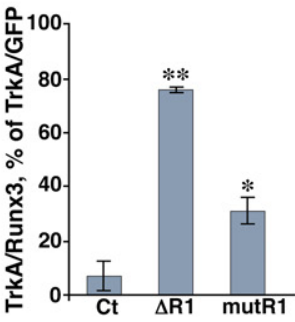

Figure 7. BRNF-binding sites are essential for R1 and R3 activity. (A) Evolutionarily conserved BRNF-binding sites within R3 core region. Red boxes mark the BRNF-binding sites identified by DiAllign TF software in five or six species using core similarity 0.75 and matrix similarity optimized. The underlined binding site, marked as BrnT, corresponds to the site identified by Dykes et al. (2010). Mutations introduced into BRNF-R3-binding sites and BrnT-binding sites, labeled as mutR3 and mutR3T, respectively, are shown below the red boxes. (B) Expression of GFP in TrkC but not TrkA neurons in E14.5 BAC transgenic embryos. (C) The percentage of TrkC neurons expressing GFP at E12.5 (white) or E14.5 (gray) in transgenic DRGs expressing intact BAC-C (Ct) and either deleted or mutated BAC-C. (*) $P<0.001$ compared with Ct. (D) An evolutionarily conserved BRNF-binding site within the R1 (BRNF-R1) core region is marked by dark green, while a conserved SORY-binding site is marked by light green. Both sites were identified by DiAllign TF software in five or six species using core similarity 1 and matrix similarity 0.9. (E) Expression of GFP by BAC-C-delR1 (left) and BAC-C-mutR1 (right) in TrkC and TrkA neurons of E14.5 transgenic embryos. $(F)$ The percentage of GFP-expressing TrkC neurons in BAC transgenics (white; left panel) and the percentage of Runx3-expressing TrkC neurons in endogenously mutated embryos (gray; right panel) at E14.5. The mean for DRGs C6-T1 is presented. $\left(^{*}\right) P<0.005$; ${ }^{(*)} P<0.0001$ compared with Ct. $(G)$ As detailed in $F$ for TrkA neurons. Bar, $50 \mu \mathrm{m}$. 
Because Runx3 expression is abolished in Brn3a-deficient mice (Eng et al. 2007), we hypothesized that, besides its impact on R3 activity, Brn3a also regulates the activity of $\mathrm{R} 1$. Inspection of the core genomic region of $\mathrm{R} 1$ for conserved TF-binding sites revealed a BRNF-R1-binding site (Fig. 7D). Mutating this binding site in either BAC-C or the mouse genome caused partial impairment of R1-enhancing activity (Fig. 7E). Specifically, mutations in the BRNF-R1-binding site of BAC-C reduced GFP expression in TrkC neurons as compared with intact BAC-C. However, the effect was significantly smaller than that upon complete R1 deletion (Fig. 7F, left panel). A similar tendency was observed in CRISPR/Cas9-mediated mutation (Fig. 7F, right panel). Interestingly, the BRNF-R1 mutation also affected the repression activity of R1 in TrkA neurons. As noted previously, Runx3 expression in TrkA neurons is mediated by $\mathrm{R} 2$ and inhibited by $\mathrm{R} 1$. Consistently, E14.5 transgenics bearing intact BAC-C did not express GFP in TrkA cells. BAC-C-mutR1 conferred Runx3 expression in $50 \%$ of TrkA neurons (Fig. 7G, left panel), whereas CRISPR-mutR1 drove Runx 3 in $30 \%$ of TrkA neurons (Fig. 7G, right panel). In both cases, the mutation had a lesser effect relative to complete removal of R1.

Together, these data indicate that BRNF-binding sites participate in the enhancive and repressive activities of R1. However, the notably milder phenotype of BRNF-R1 mutants, as compared with mutants lacking the entire $\mathrm{R} 1$, raises the possibility that additional TFs are involved in regulating $\mathrm{R} 1$ function in TrkC neurons. Indeed, RNAseq analysis revealed that besides Brn3a (Pou4f1), three other TFs-Is11, Sox11, and NeuroD1-were highly expressed in purified TrkC neurons (Supplemental Table S6). Sox11, which was shown to regulate sensory neuron development and survival (Lin et al. 2011), is an interesting candidate because R1 contains a conserved SORYbinding site (Fig. 7D). The R1 Isl1-binding site is probably of lesser importance because the loss of Isl1 hardly affects Runx3 expression (Huang et al. 1999; Quina et al. 2009; Dykes et al. 2011), while NeuroD1 emerged as a Runx3responsive gene (Supplemental Table S6), placing it downstream from Runx3. Of note, comparison of RNA-seq data from wild-type/P2 ${ }^{\mathrm{GFP}}$ and $\mathrm{P} 2^{\mathrm{GFP} / \mathrm{GFP}}$ neurons (GSE81140) (Supplemental Table S7) revealed that a group of genes previously identified as targets of Brn3a (Dykes et al. 2011) was Runx3-responsive. This overlap may indicate that those presumable Brn3a target genes are, in effect, regulated by Runx3 (Supplemental Fig. S9).

\section{Discussion}

\section{REs located 100-125 kb upstream of P2 regulate TrkC} neuron expression of Runx3

In the present study, we delineated the Runx3 gene transcriptional unit. It spans $\sim 170 \mathrm{~kb}$ of genomic DNA, encompassing the gene itself and the REs that control its spatiotemporal expression specificity in different tissues. Ten CNEs were identified in this region, three of which-R1, R2, and R3-are essential for specific Runx3 expression in TrkC neurons. These REs most likely medi- ate Runx3 expression exclusively in DRGs and TGs, as none of the other putative REs that regulate Runx3 in other tissues overlap with these TrkC-specific REs /Ghisletti et al. 2010; Nakayamada et al. 2011; Vahedi et al. 2012; Hnisz et al. 2013; McClellan et al. 2013; Ohba et al. 2015; Zhou et al. 2015; Gunnell et al. 2016).

As shown previously (Levanon et al. 2003; Sullivan et al. 2008), Runx3 functions as an important component of the ancient stretch reflex neuronal circuit. This role corresponds with the finding that the three REs collaborate with the more evolutionarily conserved promoter P2 (Levanon and Groner 2004); i.e., the three REs skip the more upstream, less conserved P1 in favor of P2. Furthermore, homozygous P2 $2^{\text {GFP/GFP }}$ mice bearing an intact P1 developed severe limb ataxia due to loss of Runx3 in early developing TrkC neurons. Thus, in TrkC neurons, unlike in other cell types, P1 does not compensate for the absence of P2 (Levanon et al. 2014). Of note, two additional P2 features - the upstream distant location of REs and the presence of an Inr motif within the promoter (Bangsow et al. 2001) - are typically found in developmentally regulated genes (Lorberbaum and Barolo 2015).

\section{Combinatorial RE cross-talk regulates Runx3 in different TrkC neuronal subpopulations}

Proprioceptive neurons consist of a highly diverse population. Accordingly, interneurons and motor neuron pools receive specialized input from selected groups of sensory neurons (Eccles et al. 1957; Mears and Frank 1997; de Nooij et al. 2013), yet little is known about proprioceptor specification. de Nooij et al. (2013) have shown that proprioceptive neurons destined to innervate distinct muscle targets differ markedly in their Etv1 TF-dependent survival and differentiation. Using single-cell RNA-seq, two proprioceptive subtypes were detected in adult mouse DRGs (Usoskin et al. 2015). It was also reported that mesenchymal signals expressed in restricted dorsoventral and proximodistal domains of the developing limb are essential for endowing muscle-type identity to two distinct proprioceptive subtypes, marked by the expression of either Cdh13, Sema5, or Crtac1 (Poliak et al. 2016).

Our analysis revealed that TrkC neurons also diverge in the pattern of Runx3 expression regulation. Differential usage of Runx3 REs defines five TrkC subpopulations in brachial DRGs. Of these, R1- and R3-dependent subpopulations dominate in C6-T1 ganglia. The R1-dependent population (Fig. 5, A1,B1,C1), which is characterized by intense Runx3 expression commencing at early embryonic stages, is the major subgroup of TrkC neurons in these ganglia. Neither R3 nor R2 could rescue these TrkC neurons upon R1 deletion. The importance of R1 is further emphasized by the observation that, in its absence $(\Delta \mathrm{R} 1)$, the precision of foot placing deteriorates. Foot-placing precision depends on proprioceptive feedback from muscle spindles (Akay et al. 2014), raising the possibility that the R1-dependent subpopulation is involved in signaling from muscle spindles. The R3-dependent population (Fig. 5, B2, C2) is smaller and develops at later embryonic stages. In this population, Runx3 expression 
was hardly detected at E12.5 and increased only later on. Deletion of R3 led to loss of the B2 and C2 subgroups but did not have a notable effect on either home cage locomotion or the beam test. However, mice with deletion of $\mathrm{R} 1$ and $\mathrm{R} 2(\Delta \mathrm{R} 1,2)$-i.e., the R3-alone mice-performed poorly in the beam test and exhibited reduced home cage locomotion. Nevertheless, these mice were not ataxic. Moreover, analysis of $\Delta \mathrm{R} 1,2$ spinal cords revealed fewer afferents, most of which did not reach the ventral zone. These observations indicate that the R3-dependent subgroup is not sufficient to support normal locomotion and precision of foot placing. In addition, R2, which is not active on its own in TrkC neurons from E12.5 onward, does affect R3 activity. However, we cannot rule out the possibility that the behavioral defects are due to changes in higher brain functions that were not assayed in this study and are secondary to the loss of spinal connectivity.

$\mathrm{R} 1$ and R3 also differ in their dependence on the POU domain TF. Mutations in BRNF-R3-binding sites led to a phenotype similar to upon deletion of the entire R3. In contrast, a mutation in the BRNF-R1-binding site had a significantly lesser effect. In both REs, this binding site is probably occupied by Brn3a, which is the major POU domain TF in E11.5 TrkC neurons. RNA-seq data analysis demonstrated a particularly high expression level of both Brn3a and Sox11. The finding that R1 has a conserved SORY-binding site raises the possibility that Brn $3 a$ and Sox11 TFs cooperate in regulating $\mathrm{R} 1$ activity in TrkC neurons. Thus, it was interesting to note that POU- and Sox-binding sites are also situated adjacently in genomic regions regulating subtype specification of dorsal spinal cord neurons (Borromeo et al. 2014). The fact that the three REs are active already at E11.5 implies that a common TF, most likely Brn3a, regulates Runx3 expression at this developmental stage. Interestingly, after E11.5, as additional TFs come into play, the relative contribution of various TFs to RE function diverges, resulting in the observed differential activity of the three REs.

The stringent cell-specific Runx3 expression observed in TrkC neurons is attained by not only the REs' enhancing capacity but also their inhibitory activity in non-TrkC neurons. The establishment of cell-type specificity through repressing expression in the irrelevant cells is a widely accepted paradigm (Lanier et al. 2009). In the developing DRGs, R1 functions as a repressor in TrkA neurons, abolishing the R2-mediated Runx3 expression in these neurons. Additionally, R2 exerts Runx3 repression in TrkB neurons, facilitating the gain of their identity. REs that function in an opposing manner to specify cell identity were also reported for other genes, including Sonic hedgehog (Shh) (Lettice et al. 2012).

The spatiotemporal specificity of developmental TFs such as Runx3 is commonly regulated by multiple REs (Lagha et al. 2012). Several modes of action have been described for gene expression regulation by multiple REs. The elements act either synergistically to increase robustness or antagonistically to enhance specificity (Andrey et al. 2013; Levine et al. 2014). Alternatively, REs may act sequentially or as shadow REs that balance genetic or environmental perturbations (Schwarzer and Spitz
2014) or else respond dynamically to various stimuli (Joo et al. 2016). At the onset of Runx3 expression, R1, R2, and R3 act synergistically but segregate later to establish the spatiotemporal expression patterns in distinct TrkC subgroups. Thus, each RE has a specific role, and none acts as a shadow enhancer. Overall, this Runx3 RE ensemble functions as an integral unit, underscoring the Heinz et al. (2015) paradigm of superenhancers controlling expression of lineage-specific genes.

Transitory Runx3 expression at E11.5 fails to rescue TrkC neurons

As noted earlier, low amounts of Runx3 were detected in E11.5 DRGs of $\Delta \mathrm{R} 1,3$ mice. However, the exclusively R2driven Runx3 expression completely disappeared at E12.5, as did the prospective TrkC neurons. Accordingly, these mice encountered a severe neonatal ataxia that recapitulated the Runx3 $3^{-/-}$phenotype (Levanon et al. 2002). This finding indicates that transient Runx3 expression commencing at E11.5 does not rescue proprioceptor development. In contrast, $\Delta \mathrm{R} 1,2$ mice expressing only low Runx3 levels at E12.5 showed a later gain of Runx3 expression and exhibited no ataxia, although they did display locomotion defects in home cage and beam tests. Given that normal locomotion requires proper function of extensor and flexor muscles, which form connections with distinct TrkC subtypes (Poliak et al. 2016), the fact that $\Delta \mathrm{R} 1,2$ mice are not ataxic supports the notion of functional heterogeneity within this R3-only TrkC neuron subgroup. Moreover, given that R3 is less conserved than R1 (Supplemental Table S2), this subgroup may be associated with a phylogenetically more recent function of TrkC neurons. A somewhat similar situation occurs in the Isl1 gene, where the less evolutionarily conserved CREST2 enhancer, which coappears with limb development, is required for a new subtype of motor neurons (Kim et al. 2015).

\section{Materials and methods}

\section{Mouse strains}

The experiments were conducted in strict accordance with the recommendations of the US National Institutes of Health Guide for the Care and Use of Laboratory Animals. The protocols were approved by the Weizmann Institute of Science Committee on the Ethics of Animal Experiments. P1 ${ }^{\mathrm{AFP}}$ and $\mathrm{P} 2^{\mathrm{GFP}}$ mice were described previously (Levanon et al. 2011). Further information regarding mouse strains is in the Supplemental Material.

\section{CNE identification}

For CNE identification, we performed sequence alignments using genomic sequences spanning the mouse Runx3 locus (chromosome $4: 134,953,991-135,208,237$; mm10) according to the University of California at Santa Cruz (UCSC) genome browser (http://genome.ucsc.edu) and the corresponding genomic regions from humans (human February 2009 assembly), rats (rat November 2004 assembly), chickens (chicken November 2011 assembly), frogs (Xenopus tropicalis November 2009 assembly), and fugu (fugu October 2011 assembly). The mouse genomic 
sequence served as the basis for sequence alignments using M-LAGAN (http://genome.lbl.gov/vista/index.shtml) (Brudno 2007).

\section{$B A C$ reporter constructs}

Six BACs were obtained from Children's Hospital Oakland Research Institute (http://bacpac.chori.org) (Supplemental Table S1). Each BAC was modified using Quick \& Easy conditional knockout kit -FRT (GeneBridges) by insertion of LacZ or EGFP in-frame into exon 3 of the murine Runx 3 at genomic position chromosome 4: 134,711,240, mm9 assembly, UCSC genome browser (http://genome.ucsc.edu).

To reduce variability, transient transgenic embryos or established BAC construct lines (at least two) were selected based on E14.5 whole-mount reporter intensity and precision in recapitulating the endogenous Runx3 expression in the skeleton, whiskers, eyelids, and nose (Levanon et al. 2001, 2011).

\section{CRISPR/Cas9-mediated in vivo deletions}

The single guided RNA (sgRNA) sequences used in this study are in Supplemental Table S4. Cas9 and sgRNA plasmids encompass the T7 promoter. sgRNA and Cas9 RNA were purified using MEGAclear kit (Life Technologies, AM 1908). sgRNAs were microinjected (2.5 $\mu$ g each of $5^{\prime}$ and $3^{\prime}$ sgRNA) together with $5 \mu \mathrm{g}$ of Cas9 RNA into fertilized mouse eggs. At least two distinct mouse lines were analyzed for each deleted or mutated RE. Further information regarding CRISPR/Cas9 deletions is included in the Supplemental Material.

\section{LacZ, GFP, and immunofluorescence analyses}

$\mathrm{X}$-Gal staining, GFP expression, and immunofluorescence analyses were performed on brachial DRG sections as described previously (Levanon et al. 2001, 2011). Further information is included in the Supplemental Material.

\section{Determination of the percentage of GFP- or Runx3-retaining TrkC neurons}

The percentage of either BAC-derived GFP-expressing neurons or Runx3 expression in CRISPR/Cas9-derived mutants was determined by monitoring TrkC, GFP, and Runx3 immunofluorescence. At least two different lines of either transgenic BAC or CRISPR/Cas9 mutants were analyzed. For each line, sagittal sections of at least four brachial-level DRGs from three to seven embryos were monitored. Percentage was calculated by dividing the number of Runx3/TrkC/GFP neurons by the total number of TrkC/GFP neurons (in CRISPR/Cas9 mutants) or by TrkC/ Runx3 neurons (in BAC transgenics).

\section{Cell number determination}

Neuronal cell counting was performed on serial transverse sections of C5-T1 brachial ganglia from two to four embryos for each genotype and from four wild-type/P2 ${ }^{\mathrm{GFP}}$ control embryos at E15.5 $(4 \mu \mathrm{m})$ and E17.5 (10 $\mu \mathrm{m})$. Given that ImagePro analysis determined the average diameter of a TrkC neuron as $\sim 19 \mu \mathrm{m}$, every fourth section of right and left ganglia was analyzed using the ImageJ software. To obtain the number of positive neurons per ganglion, the average number of positive cells per section was multiplied by the number of sections and divided by 4.75 (19 $\mu \mathrm{m}$ diameter; $4-\mu \mathrm{m}$ section). Further information regarding deter- mination of Runx3 intensity is included in the Supplemental Material. Kolmogorov-Smirnov statistical test was used for analyses of immunohistochemical data. Results obtained from lines of the same genotype were compiled based on their insignificant deviation. Data are presented throughout as mean and SEM.

\section{Behavioral studies}

Home cage locomotion was assessed on males using the InfraMot system (TSE Systems, http://www.tsesystems.com/products/ behavior/homecage/phenomaster/activity/inframot.htm). The beam-walking test was conducted using a 6-mm-wide beam as detailed in the Supplemental Material. The following indices were examined: time to cross over (in seconds), number of steps, and falls ratio (percentage $=$ number of falls/number of steps).

\section{Analysis of TF-binding sites}

Sequences of R1 and R3 were analyzed for TF-binding sites using DiAlign TF; multiple alignment and TF-binding sites (Morgenstern et al. 1998) are available at the Genomatix Web site (http ://www.genomatix.de). Further information regarding gene expression analysis is included in the Supplemental Material. RNA-seq data were deposited into the Gene Expression Omnibus repository under the accession number GSE81140.

\section{Acknowledgments}

We thank Timothy Dahlem from the University of Utah Mutation Generation and Detection Core for sgRNA constructs and advice; Joseph Lotem for stimulating discussions and helpful comments on the manuscript; Joseph Dicken and Tal Porat for help in BAC manipulations; Rafi Saka, Pavel Bell, Ofira Higfa, and Dalia Vaknin for devoted mice care; Rebecca Haffner-Krausz, Golda Damari, Alina Berkovitz, and Sima Peretz for generation of transgenic and knockout mice; and Daniela Salzenstein-Aman and Shlomit Gilad from the Grand Israel National Center for Personalized Medicine (G-INCPM) at the Weizmann Institute for RNA-seq analysis. This study was supported by a grant from the Israel Science Foundation (ISF) to D.L. and Y.G.

\section{References}

Akay T, Tourtellotte WG, Arber S, Jessell TM. 2014. Degradation of mouse locomotor pattern in the absence of proprioceptive sensory feedback. Proc Natl Acad Sci 111: 16877-16882.

Andrey G, Montavon T, Mascrez B, Gonzalez F, Noordermeer D, Leleu M, Trono D, Spitz F, Duboule D. 2013. A switch between topological domains underlies HoxD genes collinearity in mouse limbs. Science 340: 1234167.

Bangsow C, Rubins N, Glusman G, Bernstein Y, Negreanu V, Goldenberg D, Lotem J, Ben-Asher E, Lancet D, Levanon D, et al. 2001. The RUNX3 gene-sequence, structure and regulated expression. Gene 279: 221-232.

Bauer O, Sharir A, Kimura A, Hantisteanu S, Takeda S, Groner Y. 2015. Loss of osteoblast Runx3 produces severe congenital osteopenia. Mol Cell Biol 35: 1097-1109.

Borromeo MD, Meredith DM, Castro DS, Chang JC, Tung KC, Guillemot F, Johnson JE. 2014. A transcription factor network specifying inhibitory versus excitatory neurons in the dorsal spinal cord. Development 141: 2803-2812.

Brenner O, Levanon D, Negreanu V, Golubkov O, Fainaru O, Woolf E, Groner Y. 2004. Loss of Runx3 function in leukocytes 
is associated with spontaneously developed colitis and gastric mucosal hyperplasia. Proc Natl Acad Sci 101: 16016-16021.

Brudno M. 2007. An introduction to the Lagan alignment toolkit. Methods Mol Biol 395: 205-220.

Buecker C, Wysocka J. 2012. Enhancers as information integration hubs in development: lessons from genomics. Trends $\mathrm{Ge}$ net 28: 276-284.

Cannavo E, Khoueiry P, Garfield DA, Geeleher P, Zichner T, Gustafson EH, Ciglar L, Korbel JO, Furlong EE. 2016. Shadow enhancers are pervasive features of developmental regulatory networks. Curr Biol 26: 38-51.

Chen AI, de Nooij JC, Jessell TM. 2006a. Graded activity of transcription factor Runx3 specifies the laminar termination pattern of sensory axons in the developing spinal cord. Neuron 49: 395-408.

Chen CL, Broom DC, Liu Y, de Nooij JC, Li Z, Cen C, Samad OA, Jessell TM, Woolf CJ, Ma Q. 2006b. Runx1 determines nociceptive sensory neuron phenotype and is required for thermal and neuropathic pain. Neuron 49: 365-377.

Cruz-Guilloty F, Pipkin ME, Djuretic IM, Levanon D, Lotem J, Lichtenheld MG, Groner Y, Rao A. 2009. Runx3 and T-box proteins cooperate to establish the transcriptional program of effector CTLs. J Exp Med 206: 51-59.

de Nooij JC, Doobar S, Jessell TM. 2013. Etv1 inactivation reveals proprioceptor subclasses that reflect the level of NT3 expression in muscle targets. Neuron 77: 1055-1068.

Dicken J, Mildner A, Leshkowitz D, Touw IP, Hantisteanu S, Jung S, Groner Y. 2013. Transcriptional reprogramming of CD11b +Esam(hi) dendritic cell identity and function by loss of Runx3. PLoS One 8: e77490.

Djuretic IM, Levanon D, Negreanu V, Groner Y, Rao A, Ansel KM. 2007. Transcription factors T-bet and Runx3 cooperate to activate Ifng and silence Il 4 in T helper type 1 cells. Nat Immunol 8: 145-153.

Dykes IM, Lanier J, Eng SR, Turner EE. 2010. Brn3a regulates neuronal subtype specification in the trigeminal ganglion by promoting Runx expression during sensory differentiation. Neural Dev 5: 3.

Dykes IM, Tempest L, Lee SI, Turner EE. 2011. Brn3a and Islet1 act epistatically to regulate the gene expression program of sensory differentiation. J Neurosci 31: 9789-9799.

Eccles JC, Eccles RM, Lundberg A. 1957. The convergence of monosynaptic excitatory afferents on to many different species of a motoneurones. J Physiol 137: 22-50.

Eng SR, Dykes IM, Lanier J, Fedtsova N, Turner EE. 2007. POUdomain factor Brn3a regulates both distinct and common programs of gene expression in the spinal and trigeminal sensory ganglia. Neural Dev 2: 3.

Fainaru O, Woolf E, Lotem J, Yarmus M, Brenner O, Goldenberg D, Negreanu V, Bernstein Y, Levanon D, Jung S, et al. 2004. Runx3 regulates mouse TGF- $\beta$-mediated dendritic cell function and its absence results in airway inflammation. EMBO $J$ 23: 969-979.

Frazer KA, Pachter L, Poliakov A, Rubin EM, Dubchak I. 2004. VISTA: computational tools for comparative genomics. Nucleic Acids Res 32: W273-W279.

Ghisletti S, Barozzi I, Mietton F, Polletti S, De Santa F, Venturini E, Gregory L, Lonie L, Chew A, Wei CL, et al. 2010. Identification and characterization of enhancers controlling the inflammatory gene expression program in macrophages. Immunity 32: 317-328.

Gunnell A, Webb HM, Wood CD, McClellan MJ, Wichaidit B, Kempkes B, Jenner RG, Osborne C, Farrell PJ, West MJ. 2016. RUNX super-enhancer control through the Notch path- way by Epstein-Barr virus transcription factors regulates B cell growth. Nucleic Acids Res 44: 4636-4650.

Heinz S, Romanoski CE, Benner C, Glass CK. 2015. The selection and function of cell type-specific enhancers. Nat Rev Mol Cell Biol 16: 144-154.

Hnisz D, Abraham BJ, Lee TI, Lau A, Saint-Andre V, Sigova AA, Hoke HA, Young RA. 2013. Super-enhancers in the control of cell identity and disease. Cell 155: 934-947.

Huang EJ, Zang K, Schmidt A, Saulys A, Xiang M, Reichardt LF. 1999. POU domain factor Brn-3a controls the differentiation and survival of trigeminal neurons by regulating Trk receptor expression. Development 126: 2869-2882.

Inoue $\mathrm{K}$, Ozaki S, Shiga T, Ito K, Masuda T, Okado N, Iseda T, Kawaguchi S, Ogawa M, Bae SC, et al. 2002. Runx3 controls the axonal projection of proprioceptive dorsal root ganglion neurons. Nat Neurosci 5: 946-954.

Inoue K, Ito K, Osato M, Lee B, Bae SC, Ito Y. 2007. The transcription factor Runx3 represses the neurotrophin receptor TrkB during lineage commitment of dorsal root ganglion neurons. J Biol Chem 282: 24175-24184.

Joo JY, Schaukowitch K, Farbiak L, Kilaru G, Kim TK. 2016. Stimulus-specific combinatorial functionality of neuronal c-fos enhancers. Nat Neurosci 19: 75-83.

Kim N, Park C, Jeong Y, Song MR. 2015. Functional diversification of motor neuron-specific Isll enhancers during evolution. PLoS Genet 11: e1005560.

Kramer I, Sigrist M, de Nooij JC, Taniuchi I, Jessell TM, Arber S. 2006. A role for Runx transcription factor signaling in dorsal root ganglion sensory neuron diversification. Neuron 49: 379-393.

Lagha M, Bothma JP, Levine M. 2012. Mechanisms of transcriptional precision in animal development. Trends Genet 28: 409-416.

Lallemend F, Ernfors P. 2012. Molecular interactions underlying the specification of sensory neurons. Trends Neurosci 35: 373-381.

Lallemend F, Sterzenbach U, Hadjab-Lallemend S, Aquino JB, Castelo-Branco G, Sinha I, Villaescusa JC, Levanon D, Wang Y, Franck MC, et al. 2012. Positional differences of axon growth rates between sensory neurons encoded by Runx3. EMBO I 31: 3718-3729.

Lanier J, Dykes IM, Nissen S, Eng SR, Turner EE. 2009. Brn3a regulates the transition from neurogenesis to terminal differentiation and represses non-neural gene expression in the trigeminal ganglion. Dev Dyn 238: 3065-3079.

Lettice LA, Williamson I, Wiltshire JH, Peluso S, Devenney PS, Hill AE, Essafi A, Hagman J, Mort R, Grimes G, et al. 2012. Opposing functions of the ETS factor family define Shh spatial expression in limb buds and underlie polydactyly. Dev Cell 22: 459-467.

Levanon D, Groner Y. 2004. Structure and regulated expression of mammalian RUNX genes. Oncogene 23: 4211-4219.

Levanon D, Brenner O, Negreanu V, Bettoun D, Woolf E, Eilam R, Lotem J, Gat U, Otto F, Speck N, et al. 2001. Spatial and temporal expression pattern of Runx3 (Aml2) and Runx1 (Aml1) indicates non-redundant functions during mouse embryogenesis. Mech Dev 109: 413-417.

Levanon D, Bettoun D, Harris-Cerruti C, Woolf E, Negreanu V, Eilam R, Bernstein Y, Goldenberg D, Xiao C, Fliegauf M, et al. 2002. The Runx3 transcription factor regulates development and survival of TrkC dorsal root ganglia neurons. EMBO J 21: 3454-3463.

Levanon D, Glusman G, Bettoun D, Ben-Asher E, Negreanu V, Bernstein Y, Harris-Cerruti C, Brenner O, Eilam R, Lotem J, et al. 2003. Phylogenesis and regulated expression of the 
RUNT domain transcription factors RUNX1 and RUNX3. Blood Cells Mol Dis 30: 161-163.

Levanon D, Bernstein Y, Negreanu V, Bone KR, Pozner A, Eilam R, Lotem J, Brenner O, Groner Y. 2011. Absence of Runx3 expression in normal gastrointestinal epithelium calls into question its tumour suppressor function. $E M B O$ Mol Med 3: 593-604.

Levanon D, Negreanu V, Lotem J, Bone KR, Brenner O, Leshkowitz D, Groner Y. 2014. Transcription factor Runx3 regulates interleukin-15-dependent natural killer cell activation. Mol Cell Biol 34: 1158-1169.

Levine M, Cattoglio C, Tjian R. 2014. Looping back to leap forward: transcription enters a new era. Cell 157: 13-25.

Lin L, Lee VM, Wang Y, Lin JS, Sock E, Wegner M, Lei L. 2011. Sox11 regulates survival and axonal growth of embryonic sensory neurons. Dev Dyn 240: 52-64.

Lorberbaum DS, Barolo S. 2015. Enhancers: holding out for the right promoter. Curr Biol 25: R290-R293.

Lotem J, Levanon D, Negreanu V, Leshkowitz D, Friedlander G, Groner Y. 2013. Runx3-mediated transcriptional program in cytotoxic lymphocytes. PLoS One 8: e80467.

Luong TN, Carlisle HJ, Southwell A, Patterson PH. 2011. Assessment of motor balance and coordination in mice using the balance beam. J Vis Exp doi: 10.3791/2376.

McClellan MJ, Wood CD, Ojeniyi O, Cooper TJ, Kanhere A, Arvey A, Webb HM, Palermo RD, Harth-Hertle ML, Kempkes B, et al. 2013. Modulation of enhancer looping and differential gene targeting by Epstein-Barr virus transcription factors directs cellular reprogramming. PLoS Pathog 9: e1003636.

Mears SC, Frank E. 1997. Formation of specific monosynaptic connections between muscle spindle afferents and motoneurons in the mouse. J Neurosci 17: 3128-3135.

Morgenstern B, Frech K, Dress A, Werner T. 1998. DIALIGN: finding local similarities by multiple sequence alignment. Bioinformatics 14: 290-294.

Naito T, Taniuchi I. 2010. The network of transcription factors that underlie the CD4 versus CD8 lineage decision. Int Immunol 22: 791-796.

Nakamura S, Senzaki K, Yoshikawa M, Nishimura M, Inoue K, Ito Y, Ozaki S, Shiga T. 2008. Dynamic regulation of the expression of neurotrophin receptors by Runx3. Development 135: $1703-1711$.
Nakayamada S, Kanno Y, Takahashi H, Jankovic D, Lu KT, Johnson TA, Sun HW, Vahedi G, Hakim O, Handon R, et al. 2011. Early Th1 cell differentiation is marked by a Tfh cell-like transition. Immunity 35: 919-931.

Ohba S, He X, Hojo H, McMahon AP. 2015. Distinct transcriptional programs underlie Sox9 regulation of the mammalian chondrocyte. Cell Rep 12: 229-243.

Poliak S, Norovich AL, Yamagata M, Sanes JR, Jessell TM. 2016. Muscle-type identity of proprioceptors specified by spatially restricted signals from limb mesenchyme. Cell 164: 512-525.

Quina LA, Wang S, Ng L, Turner EE. 2009. Brn3a and Nurr1 mediate a gene regulatory pathway for habenula development. I Neurosci 29: 14309-14322.

Raveh E, Cohen S, Levanon D, Groner Y, Gat U. 2005. Runx3 is involved in hair shape determination. Dev Dyn 233: $1478-1487$.

Schwarzer W, Spitz F. 2014. The architecture of gene expression: integrating dispersed cis-regulatory modules into coherent regulatory domains. Curr Opin Genet Dev 27: 74-82.

Sullivan JC, Sher D, Eisenstein M, Shigesada K, Reitzel AM, Marlow H, Levanon D, Groner Y, Finnerty JR, Gat U. 2008. The evolutionary origin of the Runx/CBF $\beta$ transcription factors —studies of the most basal metazoans. BMC Evol Biol 8: 228.

Usoskin D, Furlan A, Islam S, Abdo H, Lonnerberg P, Lou D, Hjerling-Leffler J, Haeggstrom J, Kharchenko O, Kharchenko PV, et al. 2015. Unbiased classification of sensory neuron types by large-scale single-cell RNA sequencing. Nat Neurosci 18: 145-153.

Vahedi G, Takahashi H, Nakayamada S, Sun HW, Sartorelli V, Kanno Y, O'Shea JJ. 2012. STATs shape the active enhancer landscape of T cell populations. Cell 151: 981-993.

Woolf E, Xiao C, Fainaru O, Lotem J, Rosen D, Negreanu V, Bernstein Y, Goldenberg D, Brenner O, Berke G, et al. 2003. Runx3 and Runx1 are required for CD8 T cell development during thymopoiesis. Proc Natl Acad Sci 100: 7731-7736.

Yamashiro T, Aberg T, Levanon D, Groner Y, Thesleff I. 2002. Expression of Runx $1,-2$ and -3 during tooth, palate and craniofacial bone development. Gene Expr Patterns 2: 109-112.

Zhou H, Schmidt SC, Jiang S, Willox B, Bernhardt K, Liang J, Johannsen EC, Kharchenko P, Gewurz BE, Kieff E, et al. 2015. Epstein-Barr virus oncoprotein super-enhancers control B cell growth. Cell Host Microbe 17: 205-216. 


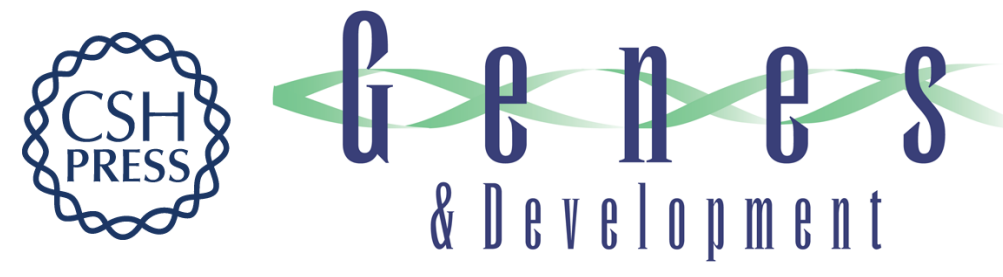

\section{An ensemble of regulatory elements controls $R$ unx3 spatiotemporal expression in subsets of dorsal root ganglia proprioceptive neurons}

Elena Appel, Sarit Weissmann, Yehuda Salzberg, et al.

Genes Dev. 2016, 30:

Access the most recent version at doi:10.1101/gad.291484.116

\section{Supplemental http://genesdev.cshlp.org/content/suppl/2016/12/19/gad.291484.116.DC1 \\ Material}

References This article cites 64 articles, 18 of which can be accessed free at:

http://genesdev.cshlp.org/content/30/23/2607.full.html\#ref-list-1

Creative This article, published in Genes \& Development, is available under a Creative Commons

Commons License (Attribution-NonCommercial 4.0 International), as described at

License http://creativecommons.org/licenses/by-nc/4.0/.

Email Alerting Receive free email alerts when new articles cite this article - sign up in the box at the top Service right corner of the article or click here.

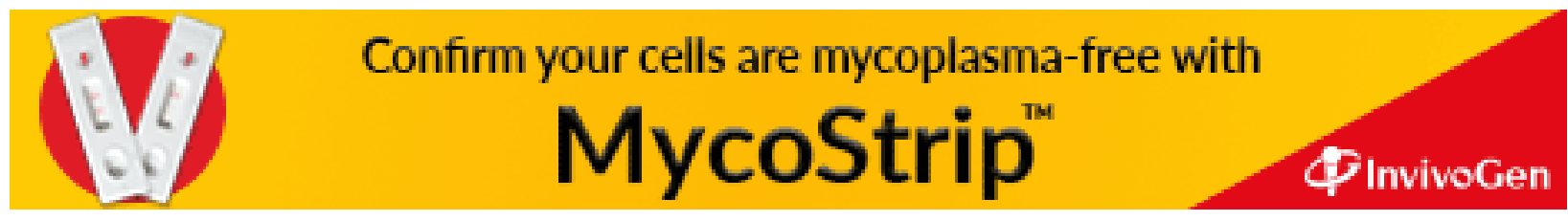

